\title{
55. PALEOCEANOGRAPHIC IMPLICATIONS OF EOCENE-OLIGOCENE CALCAREOUS NANNOFOSSILS FROM SITES 711 AND 748 IN THE INDIAN OCEAN ${ }^{1}$
}

\author{
Wuchang Wei, ${ }^{2}$ Giuliana Villa, ${ }^{3}$ and Sherwood W. Wise, Jr. ${ }^{2}$
}

\begin{abstract}
An Eocene-Oligocene calcareous nannofossil biostratigraphic framework for Ocean Drilling Program (ODP) Site 748 in the southern Indian Ocean is established, which provides a foundation for this and future quantitative biogeographic studies. This biostratigraphic analysis, together with quantitative nannofossil data, enables a reinterpretation of the preliminary magnetostratigraphy and a new placement for magnetic Subchron C13N in the lowermost Oligocene.

Calcareous nannofossil species diversity is low at Site 748 relative to lower latitude sites, with about 13 taxa in the middle Eocene, gradually decreasing to about 6 in the late Oligocene. There is, however, no apparent mass extinction at any stratigraphic level. Similarly, no mass extinctions were recorded at or near the Eocene/Oligocene boundary at Site 711 in the equatorial Indian Ocean. Species diversity at the equatorial site is significantly higher than at Site 748 , with a maximum of 39 species in the middle Eocene and a minimum of 14 species in the late Oligocene. The abundance patterns of nannofossil taxa are also quite different at the two sites, with chiasmoliths, Isthmolithus recurvus, and Reticulofenestra daviesii abundant and restricted to the high-latitude site and Coccolithus formosus, discoasters, and sphenoliths abundant at the equatorial site but impoverished at the high-latitude site. This indicates a significant latitudinal biogeographic gradient between the equatorial site and the high-latitude site in the Indian Ocean for the middle Eocene-Oligocene interval.

The abundance change of warm-water taxa is similar to that of species diversity at Site 711. There is a general trend of decreasing abundance of warm-water taxa from the middle Eocene through the early Oligocene at Site 711, suggesting a gradual cooling of the surface waters in the equatorial Indian Ocean. The abundance of warm-water taxa increased in the late Oligocene, in association with an increase in species diversity, and this may reflect a warming of the surface waters in the late Oligocene.

An abrupt increase in the abundance of cool-water taxa (from $20 \%$ to over $90 \%$ ) occurred from 36.3 to $35.9 \mathrm{Ma}$ at high-latitude Site 748 . Coincident with this event was a $\sim 1.0 \%$ positive shift in the $\delta^{18} \mathrm{O}$ value of planktonic foraminifers and the occurrence of ice-rafted debris. This abrupt change in the nannofossil population is a useful biostratigraphic event for locating the bottom of magnetic Subchron C13N in the Southern Ocean. The sharp increase in cool-water taxa coeval with a large positive shift in $\delta^{18} \mathrm{O}$ values suggests that the high-latitude surface waters drastically cooled around 36.3-35.9 $\mathrm{Ma}$. The temperature drop is estimated to be $4^{\circ} \mathrm{C}$ or more at Site 748 based on the nannofossil population change relative to the latitudinal biogeographic gradient established in the South Atlantic Ocean during previous studies. Consequently, much of the $\delta^{18} \mathrm{O}$ increase at Site 748 appears to be due to a temperature drop in the high latitudes rather than an ice-volume signal. The $\sim 0.1 \% \circ \delta^{18} \mathrm{O}$ increase not accounted for by the temperature drop is attributed to an ice-volume increase of $4.6 \times 10^{3} \mathrm{~km}^{3}$, or $20 \%$ the size of the present Antarctic ice sheet.
\end{abstract}

\section{INTRODUCTION}

The middle Eocene-Oligocene is a critical period in the developmental history of our planet. During this period, the climate cooled significantly, and the earth changed from an essentially non-ice mode into an ice mode. There were profound changes in sea level (Vail and Hardenbol, 1979; Haq et al., 1987), global oceanic circulation patterns (Kennett, 1977, 1983), the carbonate compensation depth (Heath, 1969; Berger, 1973; Van Andel et al., 1975), and marine and land biotas (Benson, 1975; Fischer and Arthur, 1977). One of the 26-m.y. cyclic extinction events was proposed by Raup and Sepkoski (1984) to fall at the Eocene/Oligocene boundary. A number of authors (e.g., Alvarez et al., 1982; Asaro et al., 1982; Ganapathy, 1982) attributed the Eocene/Oligocene extinctions to extraterrestrial causes, a hypothesis that has engendered considerable debate (Keller et al., 1983; Keller, 1986).

\footnotetext{
${ }^{1}$ Wise, S. W., Jr., Schlich, R., et al., 1992. Proc. ODP, Sci. Results, 120: College Station, TX (Ocean Drilling Program).

2 Department of Geology, Florida State University, Tallahassee, FL 32306, U.S.A.

${ }^{3}$ Istituto di Geologia, Università di Parma, Viale delle Scienze, 43100 Parma, Italy.
}

Not all of the profound changes enumerated above are well understood. Opinions differ as to the number of cooling events and their timing in the Eocene-Oligocene interval. For instance, Keller (1983a) cited evidence from planktonic foraminifer assemblages and oxygen isotopes to postulate rapid cooling events at 44-43, 41-40, 39-38, 37-36, and 31-29 Ma (see also Keller, 1983b; 1986). Not all of these cooling events have been verified by other studies. On the contrary, warming events have been observed at about 43 (Wolfe, 1971; 1978) and $38 \mathrm{Ma}$ (Haq and Lohmann, 1976). There is also no agreement on the relative magnitude of the cooling events, including the often reported Eocene/Oligocene boundary cooling. Early oxygen isotope studies suggested an $\sim 4^{\circ} \mathrm{C}$ cooling in the high latitude surface waters and the deep ocean (Shackleton and Kennett, 1975; Savin et al., 1975). Some later investigators argued that the enrichment of $\delta^{18} \mathrm{O}$ near the Eocene/Oligocene boundary was caused primarily by increased global ice volume (Matthews and Poore, 1980; Poore and Matthews, 1984a, 1984b; Keigwin and Corliss, 1986; Prentice and Matthews, 1988). The documentation of a coeval occurrence of ice-rafted debris and a large $\delta^{18} \mathrm{O}$ shift near the Eocene/Oligocene boundary at Ocean Drilling Program (ODP) Site 748 led Zachos et al. (this volume) to suggest that much of the planktonic foraminiferal $\delta^{18} \mathrm{O}$ increase was the result of an increase in ice volume and about $0.2 \% 0-0.3 \%$ of the earliest Oligocene $\delta^{18} \mathrm{O}$ increase was due to lowered temperatures. That 
would suggest about $1^{\circ} \mathrm{C}$ drop in surface-water temperature at the site. Micropaleontologic studies by Haq and Lohmann (1976) and Keller (1983a) indicated that the Eocene/Oligocene cooling is a minor one compared with other episodes in the middle Eocene-Oligocene interval. A calcareous nannofossil study of the extremely high-latitude Site 689 by Wei and Wise (1990a), however, suggested that the Eocene/Oligocene cooling in highlatitude surface waters was the most drastic and severe event during the Eocene-Oligocene interval.

Surface-water temperatures at different latitudes probably have significantly different histories. It may be misleading, therefore, to extrapolate surface-water history for the low or mid latitudes to the high latitudes, where data have been relatively rare. In this paper, we study quantitatively middle Eocene-Oligocene calcareous nannofossils from Site 748 in the high-latitude southern Indian Ocean in contrast to those from ODP Site 711 in the equatorial Indian Ocean (Fig. 1). Our objectives are to contrast the nannofossil assemblages between the equatorial site and the high-latitude site, and to infer the surface-water temperature history based on changes in nannofossil assemblages at both sites. This is the first quantitative study of Paleogene nannofossil biogeography of the Indian Ocean. As such, it lays the foundation for more detailed studies in the future. Biostratigraphic and quantitative studies of the Neogene calcareous nannofossils from Site 748 and a number of other sites in the Southern Ocean are presented in Wei and Wise (this volume, Chapters 28 and 29).

Calcareous nannofossils are the skeletal remains of calcareous nannoplankton that lived in the surface waters of the ocean. Mapping of the biogeography of modern calcareous nannoplankton (McIntyre and Bé, 1967; Okada and Honjo,
1973) has shown that their distribution patterns are closely related to the thermal structure of surface waters. This is because different species have different temperature preferences. This makes the paleobiogeography of calcareous nannofossils a useful tool in reconstructing paleotemperatures of the surface waters and a number of studies have used calcareous nannofossils successfully to indicate climatic changes (e.g., McIntyre et al., 1970; Geitzenauer, 1969; Haq et al., 1977; Wei and Wise, 1990a).

\section{MATERIAL AND METHODS}

Site 711 is located in the western equatorial Indian Ocean at $2^{\circ} 44.56^{\prime} \mathrm{S}$ and $61^{\circ} 09.78^{\prime} \mathrm{E}$ (Fig. 1) at a water depth of $4428 \mathrm{~m}$. The middle Eocene-Oligocene sedimentary interval consists of two lithologic units (Fig. 2), both of which yielded abundant and generally well-preserved calcareous nannofossils. The $82-173 \mathrm{~m}$ below seafloor (mbsf) interval consists of carbonate-rich sediments that are virtually devoid of foraminifers. The sediments are characterized as nannofossil oozes or clay-bearing nannofossil oozes that become lithified toward the bottom of this unit and turn into clay-bearing nannofossil chalks. The 173-240 mbsf interval consists of radiolarian nannofossil chalks. Carbonate content is about $70 \%-80 \%$. A few shorter intervals contain an almost pure radiolarian ooze. Age assignments for Site 711 samples analyzed in this study were based on the calcareous nannofossil biostratigraphy of Okada (1990) with minor modifications based on our observations of some datum levels, using the biomagnetostratigraphic correlation information compiled by Berggren et al. (1985) and Wei and Wise (1989).

Site $748\left(58^{\circ} 26.45^{\prime} \mathrm{S} ; 78^{\circ} 58.89^{\prime} \mathrm{E}\right)$ is located on the Southern Kerguelen Plateau (Fig. 1) in $\sim 1290 \mathrm{~m}$ of water. The upper

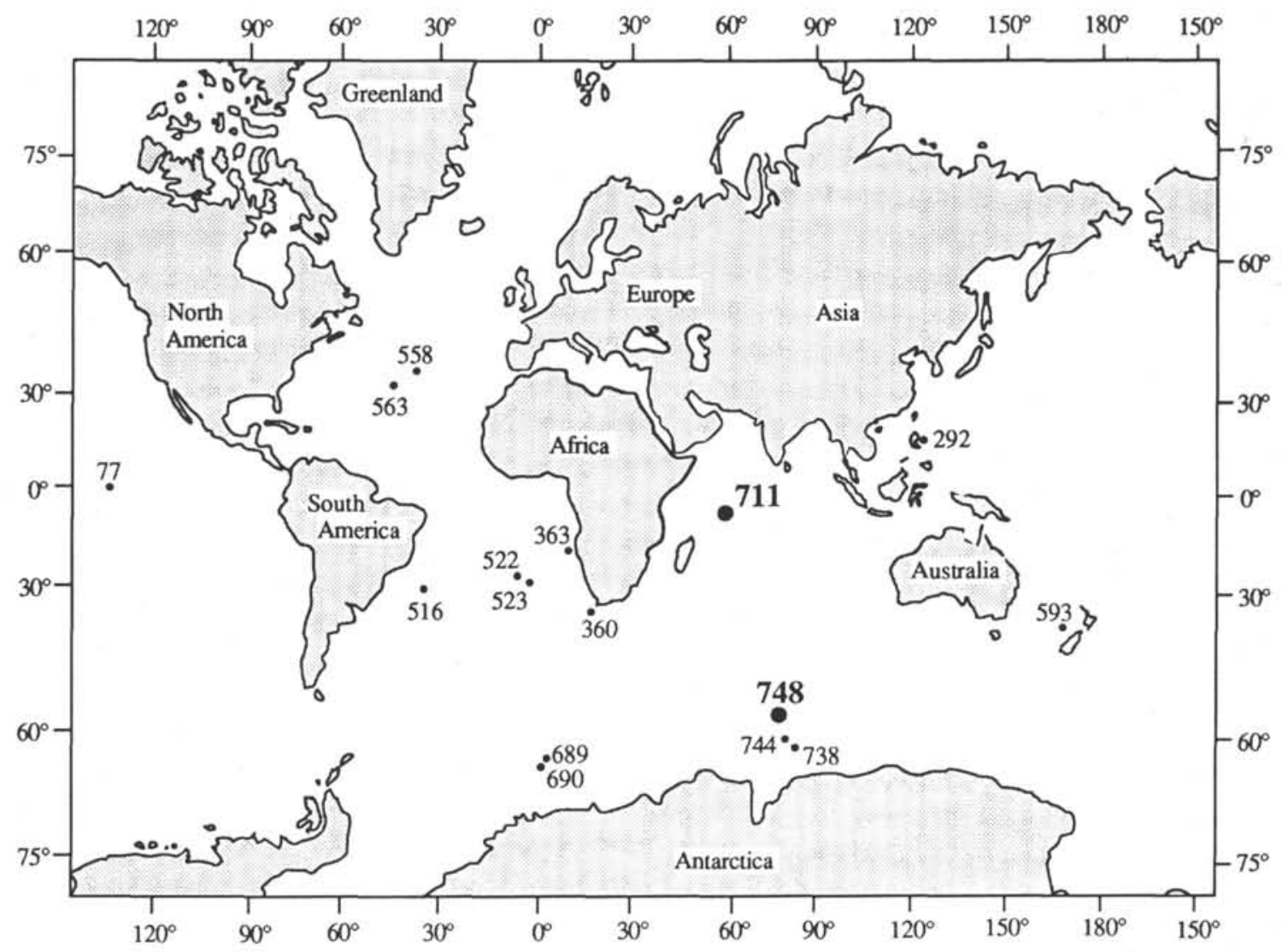

Figure 1. Locations of ODP Sites 711 and 748 in the Indian Ocean. Other DSDP/ODP sites discussed in the paper are also shown. 

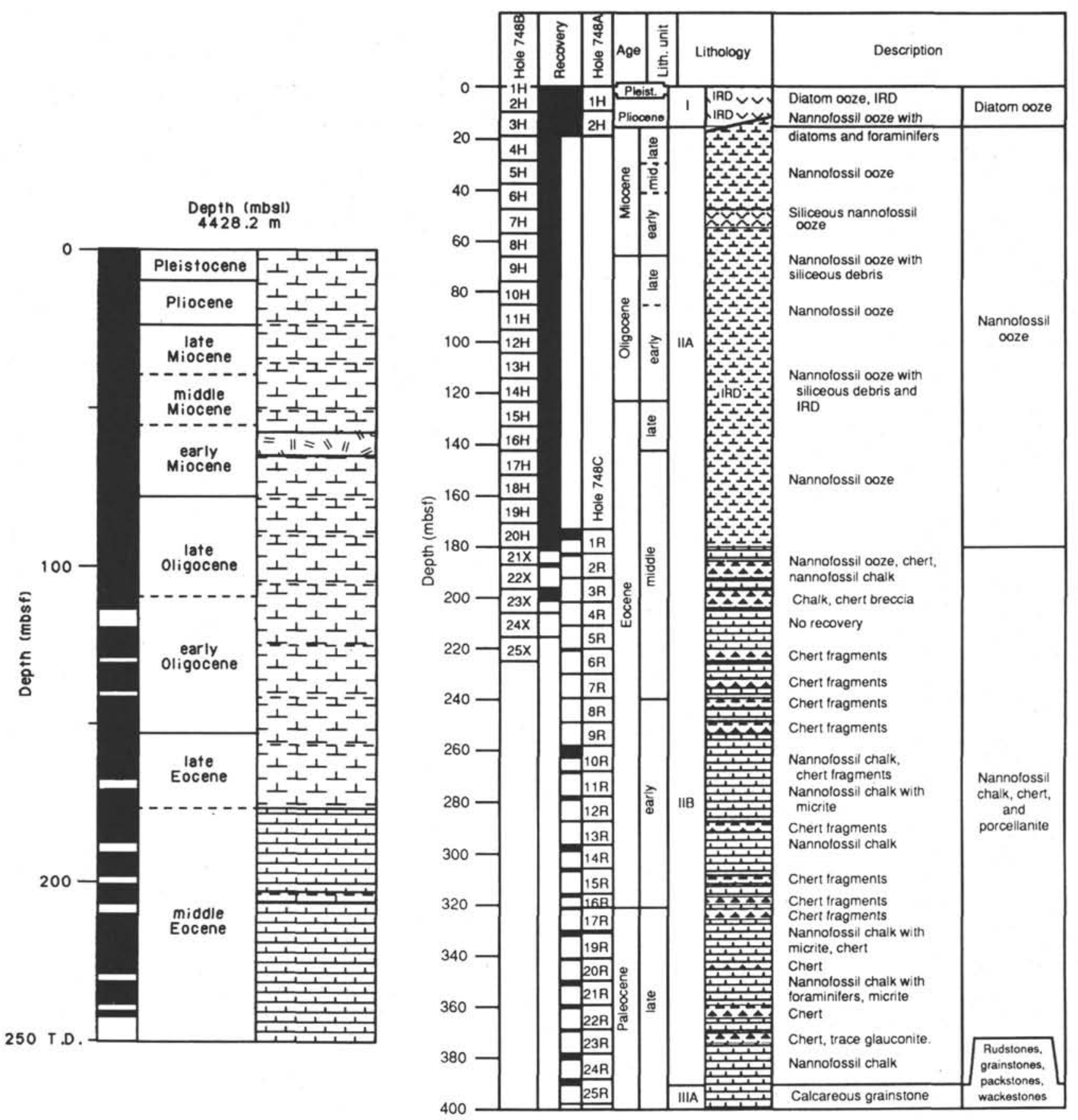

Figure 2. Stratigraphic summary of Sites 711 (left panel) and 748 (right panel). Black indicates recovered intervals.

middle Eocene through the Oligocene sequence was recovered at a rate of nearly $100 \%$. The interval is composed predominantly of nannofossil ooze with occasional influxes of diatoms or siliceous debris composed of diatoms, radiolarians, sponge spicules, and silicoflagellates (Fig. 2). Sections 120 748B-14H-1 and -14H-2 contain abundant ice-rafted debris (Breza and Wise, this volume), which are coeval with a large positive shift in the $\delta^{18} \mathrm{O}$ value of foraminifers (Zachos et al., this volume). Calcareous nannofossils are very abundant and moderately to well preserved. Paleomagnetic polarity measurements were carried out both aboard ship and in a shorebased laboratory, and the data appear to be very useful for establishing a magnetostratigraphy (Schlich, Wise, et al., 1989; Inokuchi and Heider, this volume).

To better evaluate the preliminary magnetostratigraphy (Schlich, Wise, et al., 1989) and to establish an age-depth curve for Site 748 , a prerequisite for discussing the paleoceanographic history, we first constructed a detailed nannofossil range chart. We recorded relative species abundances for one sample per core section or, in some critical intervals, multiple samples per core section, such as near the Eocene/Oligocene boundary where ice-rafted debris (Breza and Wise, this volume) and an abrupt $\delta^{18} \mathrm{O}$ excursion (Zachos et al., this volume) have been reported. 
Smear slides were made directly from unprocessed samples and were examined with a light microscope at $\times 650$ magnification. The abundance of calcareous nannofossils on each slide was estimated using the following criteria:

$$
\begin{aligned}
& \mathrm{V}=\text { very abundant }(>10 \text { specimens per field of view); } \\
& \mathrm{A}=\text { abundant }(1-10 \text { specimens per field of view); } \\
& \mathrm{C}=\text { common }(1 \text { specimen per } 2-10 \text { fields of view); } \\
& F=\text { few }(1 \text { specimen per } 11-50 \text { fields of view); } \\
& R=\text { rare }(1 \text { specimen per } 51-200 \text { fields of view); and } \\
& B=\text { barren (no specimen was found in }>200 \text { fields of view). }
\end{aligned}
$$

Preservation of the calcareous nannofossil assemblage is recorded as follows:

$\mathrm{G}=$ good (little evidence of etching or overgrowth);

$\mathbf{M}=$ moderate (etching or overgrowth is apparent);

$\mathbf{P}=$ poor (there is significant etching or overgrowth and identification of some species is impaired).

Calcareous nannofossil species considered in this paper are listed in the appendix, where they are arranged alphabetically by generic epithets. Bibliographic references for these taxa can be found in Loeblich and Tappan $(1966,1968,1969,1970 a$, 1970b, 1971, 1973), Heck (1979a, 1979b, 1980a, 1980b, 1981a, 1981b, 1982a, 1982b, 1983), or Steinmetz (1985a, 1985b, 1986, 1987a, 1987b, 1988a, 1988b, 1989).

The high-latitude nannofossil zonation of Wei and Wise $(1990 \mathrm{~b})$ is used in this study, with the erection of the last occurrence (LO) of Reticulofenestra bisecta as the marker for the Oligocene/Miocene boundary (Fig. 3). The nannofossil datums used in this zonation have been correlated with the magnetostratigraphy in the Southern Ocean at ODP Sites 689, 690 (Wei and Wise, 1990b, in press a), and 744 (Wei and Thierstein, 1991).

For the quantitative study of calcareous nannofossil assemblages from Sites 711 and 748 , about 300 specimens were counted along random traverses of each smear slide using both phase-contrast and cross-polarized light microscopy. Grouping of some taxa was necessary during our collection of the census data. The justification and detailed descriptions of the groupings have been provided in Wei and Wise (1990a) and will not be repeated here. The census data for Sites 711 and 748 are given in Tables 1 and 2, respectively.

\section{REINTERPRETATION OF THE PRELIMINARY MAGNETOSTRATIGRAPHY}

Relative abundances of calcareous nannofossil species for the middle Eocene-lowest Miocene interval at Site 748 are presented in Table 3. Stratigraphic levels of nannofossil datums at Site 748 are illustrated in Figure 4, in which the preliminary magnetostratigraphy given in Schlich, Wise, et al. (1989) is also shown. The estimated ages of some nannofossil datums based on previous studies are summarized in Table 4.

There is an abrupt increase in the abundance of cool-water taxa around 115.5 mbsf, coincident with a large $\delta^{18} \mathrm{O}$ shift (Fig. 4). A similar phenomenon was recorded at Site 689 on Maud Rise (Wei and Wise, 1990a; Stott et al., 1990; see further discussion later in this paper), where the nannofossil and isotope events occur at the bottom of magnetic Subchron C13N (SpieB, 1990), or at about 35.7-36.0 Ma using the time scale of Berggren et al. (1985). At Site 744, which is south of Site 748 , a positive $\delta^{18} \mathrm{O}$ shift of similar amplitude was recorded at the bottom of Subchron C13N (Barrera and Huber, 1991; Barron et al., 1991). Coeval with this $\delta^{18} \mathrm{O}$ shift is an abrupt increase in the abundance of cool-water nannofossil taxa (Wei, unpubl. data, 1990). The oxygen isotope excursion is also well calibrated at the bottom of magnetic Subchron $\mathrm{C} 13 \mathrm{~N}$ at mid-latitude DSDP Site 522 (Poore et al., 1984; Oberhänsli and Toumarkine, 1985) and at number of other low- or mid-latitude sites (i.e., DSDP Sites 77, 292, 563, and 593) at $35-36 \mathrm{Ma}$ as dated by biostratigraphic data (Hess et al., 1989). In other words, the oxygen isotope event has previously been found at various latitudes consistently near the bottom of Subchron $\mathrm{C} 13 \mathrm{~N}$ but never far above it. Consequently, the Subchron C13N identified in Schlich, Wise, et al. (1989) in the 118-119 mbsf interval, far below the abrupt increase in cool-water taxa and the $\delta^{18} \mathrm{O}$ shift at Site 748 , is most likely in error. This magnetic normal interval was recognized by shipboard cryogenic measurements that did not yield a consistent magnetic normal value. Furthermore, these shipboard determinations have not been supported by shorebased analysis of discrete demagnetized samples, which provide more reliable data than those determined using the whole-core, pass-through magnetometer aboard the ship. Specifically, the shore-based analyses of all the discrete samples from the middle part of Core $120-748 \mathrm{~B}-14 \mathrm{H}$ show reversed polarity (Inokuchi and Heider, this volume) rather than normal, as indicated by the shipboard results (Schlich, Wise, et al., 1989, p. 202, fig. 37). The bottom of Subchron C13N is most likely to be at 115 mbsf, nearly coincident with the abrupt increase in abundance of cool-water taxa and the $\delta^{18} \mathrm{O}$ shift.

Two calcareous nannofossil species datums also support this reinterpretation of the magnetostratigraphy. The LO of Reticulofenestra oamaruensis has a consistent age of $36.0 \mathrm{Ma}$ at Sites 689 and 744 (Fig. 5), both of which are Southern Ocean sites. The LO of Isthmolithus recurvus has an age of about 34.6 Ma at a number of mid- to high-latitude sites (Fig. 6). Moving the bottom of Subchron C13N up to 115 mbsf would give an age-depth curve that fits the magnetic subchron boundaries and the two nannofossil datums quite well (Fig. 7).

The first occurrence (FO) of Reticulofenestra reticulata is located at 171 mbsf. This datum has been found to be associated with Subchron C18R or Subchron C19N (Wei and Wise, 1989, 1990b), we interpret the magnetic normal event in the 161-162 and the 164-165 mbsf intervals as Subchron $\mathrm{C} 18 \mathrm{~N}$. This interpretation also requires minimum change in the sedimentation rates of Cores $120-748 \mathrm{~B}-18 \mathrm{H}$ and $-19 \mathrm{H}$.

Based on the above discussion, we have reinterpreted the magnetostratigraphy at Site 748 as shown in Figure 8. Inokuchi and Heider (this volume) concur with these interpretations. Age assignments for Site 748 samples analyzed in this study are based on the age-depth curve constructed using the magnetostratigraphic and nannofossil datum information given in Figure 8.

\section{EXTINCTIONS AND SPECIES DIVERSITY}

Figure 8 shows that there is a succession of calcareous nannofossil extinctions at Site 748 from the middle Eocene through the Oligocene. Several species originated in this middle Eocene-early Oligocene interval. The consequence of more extinctions than originations in this interval is a decrease of species diversity through time. There is, however, no extinction here associated with the Eocene/Oligocene boundary, which is located within the lower part of Core 120-748B$14 \mathrm{H}$. In fact, there are no more than two species extinctions at any stratigraphic level. In other words, no mass extinctions at the Eocene/Oligocene boundary or stepwise extinctions were recorded at Site 748 .

Changes in simple species diversity (number of species) from sample to sample at Sites 711 and 748 are shown in Figure 9. The data are taken from the range charts of Site 711 (Okada, 1990) and Table 3 of this report. It is clear from Figure 


\begin{tabular}{|c|l|l|l|l|}
\hline \multirow{2}{*}{ Age } & \multicolumn{1}{|c|}{$\begin{array}{c}\text { Zones used } \\
\text { in this study }\end{array}$} & Datums & $\begin{array}{c}\text { Zones of Okada } \\
\text { and Bukry (1980) }\end{array}$ & Zones of \\
Wise (1983)
\end{tabular}

Figure 3. The high-latitude zonation of Wei and Wise (1990b) used in this study with the erection of the LO of Reticulofenestra bisecta as the marker for the Oligocene/Miocene boundary, and its correlation with the zonations of Okada and Bukry (1980) and Wise (1983). 
Table 1. Age assignment of individual samples and census data of calcareous nannofossil taxa, Site 711.

\begin{tabular}{|c|c|c|c|c|c|c|c|c|c|c|c|c|c|c|c|c|c|c|}
\hline $\begin{array}{l}\text { Core, section, } \\
\text { interval }(\mathrm{cm})\end{array}$ & $\begin{array}{l}\text { Depth } \\
\text { (mbsf) }\end{array}$ & $\begin{array}{l}\text { Age } \\
\text { (Ma) }\end{array}$ & Sphe. & Disco. & $\begin{array}{l}\text { C. } \\
\text { fi. }\end{array}$ & $\begin{array}{l}\text { C. } \\
\text { pel. }\end{array}$ & $\begin{array}{l}\text { T. } \\
\text { car. }\end{array}$ & $\begin{array}{l}\text { C. } \\
\text { fen. }\end{array}$ & $\begin{array}{c}\mathrm{R} . \\
\text { bes. }\end{array}$ & $\begin{array}{l}\text { R. } \\
\text { um. }\end{array}$ & $\begin{array}{l}\text { C. } \\
\text { nit. }\end{array}$ & Heli. & $\begin{array}{l}\text { B. } \\
\text { ser. }\end{array}$ & $\begin{array}{l}\text { C. } \\
\text { for. }\end{array}$ & $\begin{array}{l}\mathbf{R} . \\
\text { ret. }\end{array}$ & $\begin{array}{l}\text { C. } \\
\text { pro. }\end{array}$ & Chias. & $\begin{array}{l}\text { P. } \\
\text { inv. }\end{array}$ \\
\hline \multicolumn{19}{|l|}{$115-711 \mathrm{~A}-$} \\
\hline $9 \mathrm{H}-6,100$ & 84.10 & 24.5 & 43.7 & 8.6 & 32.7 & 6.8 & 3.2 & 4.1 & 0.3 & 0.3 & 0.3 & 0 & 0 & 0 & 0 & 0 & 0 & 0 \\
\hline $10 \mathrm{H}-2,120$ & 88.10 & 24.8 & 43.0 & 4.2 & 38.5 & 6.5 & 3.6 & 3.2 & 0.3 & 0 & 0.6 & 0 & 0 & 0 & 0 & 0 & 0 & 0 \\
\hline $10 \mathrm{H}-4,50$ & 90.20 & 25.0 & 37.8 & 7.5 & 42.2 & 9.5 & 1.7 & 0.7 & 0.7 & 0 & 0 & 0 & 0 & 0 & 0 & 0 & 0 & 0 \\
\hline $10 \mathrm{H}-6,66$ & 93.36 & 25.3 & 32.8 & 13.4 & 39.7 & 10.8 & 3.0 & 0 & 0 & 0 & 0.3 & 0 & 0 & 0 & 0 & 0 & 0 & 0 \\
\hline $11 \mathrm{H}-3,100$ & 98.80 & 25.7 & 19.0 & 5.2 & 63.6 & 8.3 & 0.9 & 1.5 & 0 & 0 & 1.2 & 0.3 & 0 & 0 & 0 & 0 & 0 & 0 \\
\hline $11 \mathrm{H}-6,100$ & 103.30 & 26.3 & 42.5 & 5.7 & 44.1 & 4.7 & 0.3 & 1.7 & 0 & 0.3 & 0.7 & 0 & 0 & 0 & 0 & 0 & 0 & 0 \\
\hline $12 \mathrm{X}-1,118$ & 105.68 & 26.6 & 32.7 & 3.8 & 53.2 & 7.2 & 0 & 1.7 & 0 & 0.9 & 0.6 & 0 & 0 & 0 & 0 & 0 & 0 & 0 \\
\hline $12 X-3,118$ & 108.68 & 27.0 & 35.8 & 7.9 & 38.1 & 9.6 & 2.0 & 1.3 & 1.7 & 3.6 & 0 & 0 & 0 & 0 & 0 & 0 & 0 & 0 \\
\hline $13 X-2,125$ & 116.95 & 28.2 & 35.4 & 6.3 & 51.1 & 3.4 & 0 & 1.9 & 0.3 & 1.3 & 0.3 & 0 & 0 & 0 & 0 & 0 & 0 & 0 \\
\hline $13 X-5,120$ & 121.40 & 29.4 & 31.5 & 9.6 & 46.9 & 4.2 & 0.3 & 0.3 & 2.9 & 1.3 & 1.3 & 1.6 & 0 & 0 & 0 & 0 & 0 & 0 \\
\hline $13 X-6,87$ & 122.57 & 30 & 28.4 & 6.9 & 49.8 & 7.3 & 1.0 & 0.3 & 4.0 & 0.7 & 1.0 & 0.7 & 0 & 0 & 0 & 0 & 0 & 0 \\
\hline $14 \mathrm{X}-1,100$ & 124.90 & 31.7 & 18.6 & 0.7 & 68.6 & 4.2 & 0.7 & 2.3 & 2.0 & 2.0 & 0.3 & 0.3 & 0 & 0.3 & 0 & 0 & 0 & 0 \\
\hline $14 \mathrm{X}-2,101$ & 126.41 & 32.1 & 23.8 & 0.3 & 61.1 & 10.9 & 0 & 0.3 & 0.6 & 1.3 & 0.3 & 1.3 & 0 & 0 & 0 & 0 & 0 & 0 \\
\hline $14 \mathrm{X}-3,83$ & 127.73 & 33.7 & 22.0 & 3.9 & 70.1 & 3.0 & 0 & 0.7 & 0 & 0 & 0.3 & 0 & 0 & 0 & 0 & 0 & 0 & 0 \\
\hline $14 X-4,100$ & 129.40 & 34.0 & 26.5 & 0.7 & 67.8 & 2.3 & 0 & 1.7 & 0 & 0.7 & 0.3 & 0 & 0 & 0 & 0 & 0 & 0 & 0 \\
\hline $14 X-5,104$ & 130.94 & 34.2 & 25.0 & 3.1 & 60.2 & 5.7 & 0.3 & 2.6 & 0.6 & 2.6 & 0 & 0 & 0 & 0 & 0 & 0 & 0 & 0 \\
\hline $15 X-1,82$ & 132.42 & 34.3 & 31.9 & 3.3 & 37.5 & 4.3 & 0 & 0.7 & 6.9 & 15.1 & 0.3 & 0 & 0 & 0 & 0 & 0 & 0 & 0 \\
\hline $15 X-2,101$ & 136.11 & 34.4 & 31.2 & 2.0 & 42.1 & 4.6 & 1.4 & 1.4 & 4.9 & 12.3 & 0 & 0 & 0 & 0 & 0 & 0 & 0 & 0 \\
\hline $15 X-4,20$ & 138.30 & 34.5 & 32.7 & 4.4 & 31.0 & 11.2 & 0 & 5.4 & 9.5 & 5.8 & 0 & 0 & 0 & 0 & 0 & 0 & 0 & 0 \\
\hline $15 X-5,104$ & 140.40 & 34.6 & 34.2 & 4.6 & 48.0 & 6.2 & 0 & 2.2 & 1.8 & 2.5 & 0 & 0.6 & 0 & 0 & 0 & 0 & 0 & 0 \\
\hline $15 X-6,100$ & 142.10 & 34.7 & 29.2 & 4.1 & 43.1 & 11.5 & 0 & 2.0 & 7.5 & 1.7 & 0 & 0 & 0 & 0.3 & 0 & 0 & 0 & 0 \\
\hline $16 \mathrm{X}-1,82$ & 144.12 & 34.8 & 30.9 & 2.3 & 36.2 & 14.5 & 0 & 0.7 & 9.9 & 1.3 & 0 & 3.9 & 0 & 0 & 0 & 0 & 0 & 0 \\
\hline $16 \mathrm{X}-3,82$ & 147.12 & 34.9 & 28.2 & 1.6 & 37.3 & 19.2 & 0 & 1.3 & 9.4 & 0.6 & 0 & 1.0 & 0.6 & 0.3 & 0 & 0.3 & 0 & 0 \\
\hline $16 X-4,20$ & 148.00 & 35.0 & 29.3 & 3.4 & 38.4 & 10.5 & 0 & 0.7 & 12.9 & 0 & 0.7 & 3.1 & 1.0 & 0 & 0 & 0 & 0 & 0 \\
\hline $16 X-5,70$ & 150.10 & 35.1 & 21.3 & 9.6 & 31.2 & 9.6 & 0 & 1.3 & 13.6 & 4.3 & 0 & 1.7 & 2.0 & 1.7 & 0 & 2.7 & 0 & 0 \\
\hline $16 X-6,100$ & 151.80 & 35.3 & 29.8 & 2.4 & 26.5 & 13.7 & 0 & 0.6 & 14.0 & 1.2 & 0 & 8.6 & 1.2 & 1. & 0 & 0 & 0.3 & 0 \\
\hline $17 X-1,120$ & 154.20 & 35.6 & 18.8 & 1.9 & 45.9 & 12.4 & 0 & 1.9 & 15.0 & 2.5 & 0.3 & 0 & 0 & 1.3 & 0 & 0 & 0 & 0 \\
\hline $17 \mathrm{X}-2,82$ & 155.32 & 35.8 & 14.6 & 2.7 & 41.7 & 11.5 & 0 & 1.4 & 18.3 & 5.4 & 0 & 0.3 & 0 & 3.4 & 0 & 0.3 & 0 & 0 \\
\hline $17 X-3,110$ & 157.10 & 36.2 & 11.0 & 2.0 & 34.3 & 10.2 & 0 & 0.3 & 14.8 & 25.0 & 0 & 0.6 & 0 & 0.9 & 0 & 0.6 & 0.3 & 0 \\
\hline $17 X-4,120$ & 158.70 & 36.4 & 7.6 & 14.0 & 1.3 & 25.2 & 0 & 0.3 & 6.0 & 40.9 & 0 & 0 & 0 & 3 & 0 & 0.7 & 0.3 & 0 \\
\hline $17 X-5,83$ & 159.83 & 37.6 & 11.4 & 17.1 & 44.0 & 14.7 & 0 & 0.3 & 4.5 & 0.9 & 0 & 0.6 & 0 & 3.9 & 2.4 & 0 & 0 & 0 \\
\hline $17 X-6,83$ & 161.33 & 37.8 & 6.4 & 18.1 & 30.4 & 16.0 & 0 & 0 & 3.1 & 1.2 & 0 & 0 & 0.9 & 3.1 & 19.3 & 1.2 & 0 & 0 \\
\hline $18 \mathrm{X}-1,83$ & 163.53 & 38.0 & 9.7 & 24.2 & 20.1 & 14.8 & 0 & 0.2 & 6.8 & 0 & 0 & 0 & 0.5 & 3.4 & 15. & 5.3 & 0 & 0 \\
\hline $18 \mathrm{X}-2,131$ & 165.33 & 38.4 & 6.7 & 13.2 & 33.4 & 8.3 & 0 & 0.3 & 12.6 & 0.3 & 0 & 0.3 & 0.9 & 2.5 & 17.2 & 4.3 & 0 & 0 \\
\hline $18 X-4,82$ & 168.02 & 38.8 & 8.6 & 24.8 & 28.5 & 8.9 & 0 & 0 & 12.3 & 0.3 & 0 & 0 & 0 & 1.3 & 11.6 & 3.6 & 0 & 0 \\
\hline $19 \mathrm{X}-1,30$ & 172.70 & 39.1 & 12.1 & 16.8 & 32.2 & 8.4 & 0 & 0 & 11.1 & 10.1 & 0 & 0.7 & 0. & 1.7 & 0 & 6.7 & 0 & 0 \\
\hline $19 X-2,145$ & 175.35 & 39.4 & 17.9 & 18.9 & 23.2 & 6.3 & 0 & 0 & 8.1 & 3.5 & 0 & 0 & 0. & 3.2 & 13.3 & 4.9 & 0 & 0 \\
\hline $19 X-3,60$ & 176.00 & 39.7 & 15.8 & 16.1 & 20.9 & 11.1 & 0 & 0 & 11.1 & 2.2 & 0 & 0 & 0.3 & 4.7 & 13.9 & 3.8 & 0 & 0 \\
\hline $19 \times-5,92$ & 179.32 & 40.0 & 74 & 14.5 & 29.7 & 10.1 & 0 & 0 & 18.6 & 0.7 & 0 & 0 & 0.7 & 27 & 11.8 & 3.7 & 0 & 0 \\
\hline $20 \mathrm{X}-1,57$ & 182.67 & 40.2 & 12.9 & 20.7 & 30.7 & 10.4 & 0 & 0 & 21.0 & 1.0 & 0 & 0 & 1.0 & 0 & 0 & 0.6 & 0.6 & 0 \\
\hline $20 \times-4,110$ & 18770 & 40.5 & 27.9 & 11.8 & 31.0 & 19.2 & 0 & 0 & 0 & 2.4 & 0 & 0.7 & 0. & 3 & 0.3 & 1.4 & 1.0 & 0 \\
\hline $21 X-1,100$ & 192.70 & 40.8 & 33.7 & 5.5 & 32.5 & 11.7 & 0 & 0 & 0 & 7.7 & 0 & 0 & 1.2 & 5 & 0 & 0.9 & 1.2 & 0 \\
\hline $21 X-3,100$ & 194.50 & 41.0 & 34.8 & 10.3 & 22.9 & 11.3 & 0 & 0 & 0 & 10.7 & 0 & 0 & 2.2 & 3 & 0 & 2.5 & 0.9 & 0 \\
\hline $21 X-5,20$ & 197.90 & 41.3 & 26.4 & 8.4 & 40.8 & 11.0 & 0 & 1.7 & 0 & 4.7 & 0 & 1.0 & 0.3 & 3.7 & 0 & 1.3 & 0 & 0 \\
\hline $21 X-5,105$ & 198.75 & 41.6 & 39.1 & 11.7 & 28.1 & 10.7 & 0 & 0 & 0 & 2 & 0 & 0. & 0.7 & 3.0 & 0 & 0 & 1.7 & 1.3 \\
\hline $22 \mathrm{X}-2,93$ & 203.83 & 42.8 & 28.8 & 4.3 & 51.7 & 4.3 & 0 & 0.9 & 0 & 0. & 0 & 1. & 0.9 & 4.0 & 0 & 0.9 & 0.6 & 0 \\
\hline -4. 20 & 206.1 & 43. & 27.4 & 11.3 & 34.6 & 13.0 & 0 & 0 & 0 & & 0 & 1. & 0 & 5 & 0 & 1.4 & 1.4 & 0.7 \\
\hline $23 \mathrm{X}-1,100$ & 212.10 & 45.0 & 35.8 & 8.5 & 33.2 & 11.4 & 0 & 0.3 & 0 & 0. & 0 & 0. & 0 & 1. & 0 & 2.3 & 2.0 & 3.3 \\
\hline & 215.2 & 46. & 46.8 & $s$ & 14.7 & 9. & 0 & 0.3 & 0 & 7. & 0 & 0. & 0 & 6. & 0 & 0.3 & 1.7 & 5.8 \\
\hline $23 X-6,1$ & 219.72 & 46.9 & 24.6 & 3.6 & 43.6 & 8. & 0 & 0 & 0 & 7.1 & 0 & 0. & 0.3 & 2.7 & 0 & 1.5 & 1.5 & 5.6 \\
\hline $24 \mathrm{X}$ - & 221. & 47. & 56.8 & 4. & 13. & 3. & 0 & 0 & 0 & 3.2 & 0 & 0.6 & 0 & 1. & 0 & 1.5 & 2.4 & 11.5 \\
\hline $24 X-2,1$ & 223.82 & 47.1 & 38.7 & 2.9 & 34.5 & 6.1 & 0 & 0 & 0 & 0 & 0 & 0 & 0 & 0. & 0 & 0.6 & 4.2 & 12.3 \\
\hline & 2 & 47. & 18.7 & 4. & 62 & 1. & 0 & 0 & 0 & 1. & 0 & 0. & 0.3 & 1. & 0 & 0.3 & 5 & 4.3 \\
\hline $24 X-5,88$ & 227.58 & 47.3 & 43.8 & 8.8 & 30.8 & 1.9 & 0 & 0 & 0 & 0. & 0 & 0.3 & 0.3 & 1. & 0 & 0.6 & 5.5 & 3.9 \\
\hline 25 & 2 & 48. & 41 & 9. & 34. & 1.9 & 0 & 0 & 0 & 3. & 0 & 0.3 & 0.3 & 0.6 & 0 & 0.6 & 2.8 & 4.1 \\
\hline 128 & 234.58 & 48.3 & 50.2 & 9.7 & 19.1 & 3.4 & 0 & 0 & 0 & 6.0 & 0 & 0 & 0 & 6.3 & 0 & 0 & 0.6 & 4.1 \\
\hline 25 & 236. & 48.7 & 38.3 & 13.4 & 28. & 3. & 0 & 0 & 0 & 4.0 & 0 & 0 & 0 & 1.4 & 0 & 1.4 & 3.6 & 5.4 \\
\hline $25 X-5,42$ & 236.72 & 49.0 & 34.9 & 15.7 & 29.2 & 5.8 & 0 & 0 & 0 & 4.2 & 0 & 0.3 & 0 & 0 & 0 & 0 & 3.2 . & 6.4 \\
\hline
\end{tabular}

Notes: Values are expressed as a percentage of the total number of individuals counted; mbsf = meters below seafloor; ages are given using the time scale of Berggren et al. (1985). Sphe. = sphenoliths; Disco. = discoasters; C.fl. = Cyclicargolithus floridanus; C. pel. $=$ Coccolithus pelagicus; T.car. $=$ Triquetrorhabdulus carinatus; C.fen. = Clausicoccus fenestratus; R.bis. = Reticulofenestra bisecta $;$ R.um. $=$ Reticulofenestra umbilicalR samodurovii $;$ C. nit. $=$ Coronocyclus nitescens; Heli. $=$ helicosphaerids; B.ser. $=$ Bramletteius serraculoides $;$ C.for. $=$ Coccolithus formosus $;$ R.ret.

$=$ Reticulofenestra reticulata; $\mathrm{C}$.pro. = Calcidiscus protoannulus; Chias. = chiasmoliths; and $\mathrm{P}$. inv. $=$ Pseudotriquetrorhabdulus inversus .

9 that species diversity is generally more than twice as high at equatorial Site 711 than at high latitude Site 748 , suggesting a significant latitudinal biogeographic gradient during the middle Eocene-Oligocene interval.

Species diversity at both sites decreased from the middle Eocene to the early Oligocene, but no abrupt decrease in species diversity occurred at the Eocene/Oligocene boundary at either site. A large drop in species diversity, however, was recorded in the 35-34 Ma interval at Site 711, where
Coccolithus formosus, Bramletteius serraculoides, Helicosphaera perch-nielsenae, and Helicosphaera wilcoxonii disappeared (Okada, 1990). Species diversity increased slightly in the late Oligocene. At Site 748 species diversity decreased gradually from the middle Eocene through the Oligocene. This is because most of the species at the site were already cool- or cold-water forms, and they could survive better than warm-water species for any further drops in water temperature. 
Table 2. Age assignment of individual samples and census data of calcareous nannofossil taxa, Site 748 .

\begin{tabular}{|c|c|c|c|c|c|c|c|c|c|c|c|c|c|c|c|}
\hline $\begin{array}{l}\text { Core, section, } \\
\text { interval }(\mathrm{cm})\end{array}$ & $\begin{array}{l}\text { Depth } \\
\text { (mbsf) }\end{array}$ & $\begin{array}{l}\text { Age } \\
\text { (Ma) }\end{array}$ & Chia. & $\begin{array}{l}\text { C. } \\
\text { fen. }\end{array}$ & $\begin{array}{l}\text { C. } \\
\text { for. }\end{array}$ & $\begin{array}{l}\text { C. } \\
\text { pel. }\end{array}$ & $\begin{array}{l}\text { C. } \\
\text { flo. }\end{array}$ & Disc. & Helo. & $\begin{array}{l}\text { I. } \\
\text { rec. }\end{array}$ & $\begin{array}{l}\text { R. } \\
\text { bis. }\end{array}$ & $\begin{array}{l}R . \\
\text { dav. }\end{array}$ & $\begin{array}{l}\text { R. } \\
\text { ret. }\end{array}$ & $\begin{array}{c}\text { R. } \\
\text { umb. }\end{array}$ & Sphe. \\
\hline \multicolumn{16}{|l|}{$120-748 \mathrm{~A}-$} \\
\hline $10 \mathrm{H}-1,10-11$ & 76.2 & 26.9 & 18.4 & 0 & 0 & 5.6 & 6.7 & 0 & 0 & 0 & 3.7 & 65.5 & 0 & 0 & 0 \\
\hline $10 \mathrm{H}-2,10-11$ & 77.7 & 27.6 & 16.7 & 0 & 0 & 8.3 & 14.0 & 0 & 0 & 0 & 0.7 & 60.3 & 0 & 0 & 0 \\
\hline $10 \mathrm{H}-3,10-11$ & 79.2 & 28.1 & 25.8 & 0 & 0 & 6.4 & 23.1 & 0 & 0 & 0 & 1.3 & 42.8 & 0 & 0.7 & 0 \\
\hline $10 \mathrm{H}-4,10-11$ & 80.7 & 28.3 & 21.1 & 0 & 0 & 3.2 & 45.7 & 0 & 0 & 0 & 0 & 30.0 & 0 & 0 & 0 \\
\hline $10 \mathrm{H}-5,10-11$ & 82.2 & 28.4 & 25.1 & 0 & 0 & 8.5 & 1.2 & 0 & 0 & 0 & 3.8 & 60.6 & 0 & 0.9 & 0 \\
\hline $10 \mathrm{H}-6,10-11$ & 83.7 & 28.7 & 20.0 & 0 & 0 & 25.7 & 1.0 & 0 & 0 & 0 & 2.0 & 51.4 & 0 & 0 & 0 \\
\hline $11 \mathrm{H}-2,10-11$ & 87.2 & 29.1 & 33.0 & 0 & 0 & 4.3 & 1.2 & 0 & 0 & 0 & 0.3 & 60.8 & 0 & 0.3 & 0 \\
\hline $11 \mathrm{H}-3,10-11$ & 88.7 & 29.3 & 44.4 & 0 & 0 & 4.0 & 21.9 & 0 & 0 & 0 & 0.6 & 28.7 & 0 & 0.3 & 0 \\
\hline $11 \mathrm{H}-4,10-11$ & 90.2 & 29.7 & 30.6 & $\mathbf{0}$ & 0 & 1.3 & 23.1 & 0 & 0 & 0 & 0.6 & 43.4 & 0 & 0.9 & 0 \\
\hline $11 \mathrm{H}-5,10-11$ & 91.7 & 29.8 & 30.2 & 0 & 0 & 5.6 & 9.5 & 0 & 0 & 0 & 1.2 & 53.6 & 0 & 0 & 0 \\
\hline $11 \mathrm{H}-6,10-11$ & 93.2 & 29.9 & 21.9 & 0 & 0 & 9.1 & 43.0 & 0 & 0 & 0 & 2.3 & 23.4 & 0 & 0.3 & 0 \\
\hline $11 \mathrm{H}-7,10-11$ & 94.7 & 30.0 & 34.9 & 0 & 0 & 12.8 & 22.4 & 0 & 0 & 0 & 6.5 & 23.4 & $\mathbf{0}$ & 0 & 0 \\
\hline $12 \mathrm{H}-2,10-11$ & 96.7 & 31.4 & 24.7 & 0 & 0 & 11.8 & 8.0 & 0 & 0 & 0 & 0 & 55.4 & 0 & 0 & 0 \\
\hline $12 \mathrm{H}-3,10-11$ & 98.2 & 32.0 & 27.2 & 0 & 0 & 19.5 & 3.2 & 0 & 0 & 0 & 1.0 & 49.2 & 0 & 0 & 0 \\
\hline $12 \mathrm{H}-4,10-11$ & 99.7 & 32.1 & 10.4 & 0 & 0 & 11.3 & 6.0 & 0 & 0 & 0 & 3.1 & 69.2 & 0 & 0 & 0 \\
\hline $12 \mathrm{H}-5,10-11$ & 101.2 & 32.3 & 31.8 & 0 & 0 & 4.8 & 14.0 & 0 & 0 & 0 & 0 & 49.3 & 0 & 0 & 0 \\
\hline $12 \mathrm{H}-6,10-11$ & 102.7 & 32.6 & 36.3 & 0 & 0 & 5.4 & 19.3 & 0 & 0 & 0 & 0 & 39.0 & 0 & 0 & 0 \\
\hline $13 \mathrm{H}-1,10-11$ & 104.7 & 32.9 & 23.2 & 0 & 0 & 3.8 & 2.3 & 0 & $\mathbf{0}$ & 0 & 0.3 & 70.1 & $\mathbf{0}$ & 0.3 & 0 \\
\hline $13 \mathrm{H}-2,10-11$ & 106.2 & 33.0 & 32.2 & 0 & 0 & 22.2 & 0.9 & 0 & 0 & 0 & 1.2 & 40.4 & 0 & 3.2 & 0 \\
\hline $13 \mathrm{H}-3,10-11$ & 107.7 & 33.6 & 28.4 & 0 & 0 & 13.2 & 0 & 0 & 0 & 0 & 0.4 & 56.3 & 0 & 1.8 & 0 \\
\hline $13 \mathrm{H}-4,10-11$ & 109.2 & 34.1 & 26.0 & 0 & 0 & 6.2 & 0.2 & 0 & 0 & 0 & 0 & 66.0 & 0 & 1.6 & 0 \\
\hline $13 \mathrm{H}-6,10-11$ & 112.2 & 34.8 & 18.4 & 0 & 0 & 15.4 & 0 & 0 & 0 & 3.2 & 0 & 47.1 & 0 & 16.0 & 0 \\
\hline $14 \mathrm{H}-1,10-11$ & 114.2 & 35.5 & 13.6 & 0 & 0 & 6.8 & 0 & 0 & 0 & 3.1 & 2.3 & 62.6 & 0 & 11.6 & 0 \\
\hline $14 \mathrm{H}-1,66-67$ & 115.1 & 35.8 & 10.0 & 0 & 0 & 4.0 & 0 & 0 & 0 & 2.6 & 0.3 & 76.2 & 0 & 6.9 & 0 \\
\hline $14 \mathrm{H}-1,104-106$ & 115.5 & 35.9 & 6.6 & 0 & 0 & 6.6 & 0 & 0 & 0 & 1.8 & 0.3 & 82.8 & 0 & 1.8 & 0 \\
\hline $14 \mathrm{H}-1,145-146$ & 115.6 & 35.9 & 5.6 & 0 & 0 & 6.9 & 0 & 0 & 0 & 1.3 & 0 & 83.2 & 0 & 3.0 & 0 \\
\hline $14 \mathrm{H}-2,7-8$ & 115.7 & 36.0 & 46.3 & 0 & 0 & 21.4 & 0 & 0 & 0 & 0.7 & 4.3 & 10.0 & 0 & 17.4 & 0 \\
\hline $14 \mathrm{H}-2,24-25$ & 115.8 & 36.0 & 17.9 & 0 & 0 & 55.3 & 0 & 0 & 0 & 0.7 & 1.7 & 18.9 & 0 & 5.6 & 0 \\
\hline $14 \mathrm{H}-2,35-36$ & 116.0 & 36.1 & 33.5 & 0 & 0 & 48.6 & 0 & 0 & 0 & 1.7 & 2.9 & 4.9 & 0 & 8.4 & 0 \\
\hline $14 \mathrm{H}-2,54-55$ & 116.1 & 36.2 & 33.7 & 0 & 0 & 48.0 & 0 & 0 & 0 & 2.1 & 3.9 & 4.4 & 0 & 7.8 & 0 \\
\hline $14 \mathrm{H}-3,9-10$ & 117.2 & 36.3 & 10 & 0 & 0 & 78.0 & 0 & 0 & 0 & 0.6 & 0.3 & 4.1 & 0 & 7.0 & 0 \\
\hline $14 \mathrm{H}-4,9-10$ & 118.7 & 36.7 & 14.9 & 0 & 0 & 38.4 & 0 & 0 & 0 & 0.7 & 2.0 & 3.6 & 0 & 40.4 & 0 \\
\hline $14 \mathrm{H}-5,9-10$ & 120.2 & 37.0 & 31.7 & 0 & 0 & 41.9 & 0 & 0 & 0 & 1.3 & 2.0 & 4.6 & 0 & 18.5 & 0 \\
\hline $14 \mathrm{H}-6,9-10$ & 121.7 & 37.4 & 6.9 & 0 & 0 & 24.9 & 0 & 0 & 0 & 2.3 & 2.9 & 35.5 & 0 & 27.5 & 0 \\
\hline $14 \mathrm{H}-7,9-10$ & 123.2 & 37.8 & 17.7 & 0 & 0 & 30.9 & 0 & 0 & 0 & 6.4 & 7.1 & 13.8 & 0 & 24.1 & 0 \\
\hline $15 \mathrm{H}-1,10-11$ & 123.7 & 37.9 & 0 & 0 & 0 & 51.2 & 0 & 0 & 0 & 5.9 & 4.3 & 13.9 & 0 & 24.8 & 0 \\
\hline $15 \mathrm{H}-2,10-11$ & 125.2 & 38.2 & 1.2 & 0 & 0 & 51.9 & 0 & 0 & 0 & 2.8 & 3.4 & 20.7 & 0 & 20.1 & 0 \\
\hline $15 \mathrm{H}-4,10-11$ & 128.2 & 38.5 & 34.8 & 0 & 0 & 37.2 & 0 & 0 & 0 & 0 & 4.1 & 15.5 & 0 & 8.3 & 0 \\
\hline $15 \mathrm{H}-6,10-11$ & 131.2 & 38.8 & 18.9 & 0 & 0 & 37.1 & 0 & 0 & 0 & 0 & 20.1 & 2.8 & 9.7 & 11.3 & 0 \\
\hline $16 \mathrm{H}-2,10-11$ & 134.7 & 39.0 & 3.8 & 0 & 0 & 17.9 & 0 & 0 & 0 & 0 & 42.9 & 1.3 & 7.1 & 26.9 & 0 \\
\hline $16 \mathrm{H}-4,10-11$ & 137.7 & 39.4 & 11.5 & $\mathbf{0}$ & 0 & 34.8 & 0 & 0 & 0 & 0 & 11.5 & 2.5 & 31.1 & 8.6 & 0 \\
\hline $16 \mathrm{H}-6,10-11$ & 140.7 & 40.0 & 9.8 & 0 & 0 & 22.5 & 0 & 0 & 0 & 0 & 7.4 & 0.4 & 57.9 & 2.1 & 0 \\
\hline $17 \mathrm{H}-1,10-11$ & 142.7 & 40.2 & 11.7 & 0 & 0 & 19.6 & 0 & 0 & 0 & 0 & 0 & 0 & 67.0 & 1.7 & 0 \\
\hline $17 \mathrm{H}-3,10-11$ & 145.7 & 40.5 & 1.6 & 0 & 0 & 46.3 & 0 & 0 & 0 & 0 & 0 & 0 & 45.5 & 5.5 & 1.2 \\
\hline $17 \mathrm{H}-5,10-11$ & 148.7 & 40.8 & 3.9 & $\mathbf{0}$ & 0 & 49.3 & 0 & 0 & 0 & 0 & 0 & 0 & 44.6 & 2.1 & 0 \\
\hline $18 \mathrm{H}-1,10-11$ & 152.2 & 41.1 & 1.0 & 0 & 0 & 55.1 & 0 & 0 & 0 & 0 & 0 & 0 & 32.4 & 11.1 & 0.3 \\
\hline $18 \mathrm{H}-3,10-11$ & 155.2 & 41.6 & 16.6 & $\mathbf{0}$ & 0 & 59.9 & 0 & 0 & 0 & 0 & 0 & 5.6 & 6.9 & 10 & 0.9 \\
\hline $18 \mathrm{H}-5,10-11$ & 158.2 & 42.0 & 22.5 & 0 & 2.1 & 44.4 & 0 & 0 & 0 & 0 & 0 & 8.1 & 6.0 & 16.9 & 0 \\
\hline $19 \mathrm{H}-1,10-11$ & 161.7 & 42.3 & 26.1 & 0 & 2.6 & 33.9 & 0 & 0 & 0 & 0 & 0 & 11.3 & 2.6 & 23.5 & 0 \\
\hline $19 \mathrm{H}-3,10-11$ & 164.7 & 42.8 & 11.4 & 0 & 1.1 & 63.7 & 0 & 0.4 & 0 & 0 & 0 & 5.9 & 2.6 & 15.0 & 0 \\
\hline $19 \mathrm{H}-5,10-11$ & 167.7 & 42.9 & 13.7 & 0 & 2.7 & 58.0 & 0 & 5.8 & 0 & 0 & 0 & 4.9 & 3.1 & 11.9 & 0 \\
\hline $20 \mathrm{H}-1,10-11$ & 171.2 & 43.0 & 41.4 & 0 & 1.3 & 43.4 & 0 & 0.7 & 0 & 0 & 0 & 5.9 & 0 & 7.2 & 0 \\
\hline $20 \mathrm{H}-3,10-11$ & 174.2 & 43.2 & 24.1 & 0 & 5.6 & 55.4 & 0 & 2.4 & 0 & 0 & 0 & 3.2 & 0 & 9.2 & 0 \\
\hline $20 \mathrm{H}-5,10-11$ & 177.2 & 43.3 & 21.9 & 0 & 6.0 & 55.6 & 0 & 4.6 & 0 & 0 & 0 & 0 & 0 & 11.9 & 0 \\
\hline
\end{tabular}

Notes: Values are expressed as a percentage of the total number of individuals counted; mbsf = meters below seafloor; ages are given using the time scale of Berggren et al. (1985). Chia. = chiasmoliths; C.fen. = Clausicoccus fenestratus; C.for. = Coccolithus formosus; C.pel. = Coccolithus pelagicus; C.fl. = Cyclicargolithus floridanus; Disc. = discoasters; Heli. = helicosphaerids; I.rec. $=$ Isthmolithus recurvus; R.bis. $=$ Reticulofenestra bisecta; R.dav. = Reticulofenestra daviesii; R.ret. = Reticulofenestra reticulata; R.umb. = Reticulofenestra umbilica/R. samodurovii; and Sphe. $=$ sphenoliths.

\section{ABUNDANCE PATTERNS OF NANNOFOSSIL TAXA}

Abundance patterns of nannofossil taxa at Sites 711 and 748 are graphically presented in Figures 10 and 11, respectively. Coccolithus formosus, discoasters, and sphenoliths were recorded only in the middle Eocene at high-latitude Site 748 , whereas these taxa are abundant throughout much of the Eocene and Oligocene at equatorial Site 711. These results agree with those of Wei and Wise (1990a), who showed through their South Atlantic latitudinal transect that these taxa increase in abundance toward lower latitudes and thus can be classified as warm-water taxa. On the other hand,
Reticulofenestra daviesii is abundant at Site 748, especially in the Oligocene, whereas this species is absent at Site 711. Isthmolithus recurvus is common at Site 748 , but it is not recorded at Site 711. Chiasmoliths are abundant at Site 748, but their abundance is greatly reduced at Site 711 . These taxa have also been shown to increase in abundance toward higher latitudes in the South Atlantic and can be classified as coolwater taxa (Wei and Wise, 1990a).

It is notable that Reticulofenestra bisecta first occurred at $\sim 40.5 \mathrm{Ma}$ at both equatorial Site 711 and high-latitude Site 748. However, this species first occurred earlier at midlatitude sites, such as DSDP Sites 360,516 , and 523, at about 
Table 3. Distribution of middle Eocene-lowest Miocene calcareous nannofossils, Hole 748B.

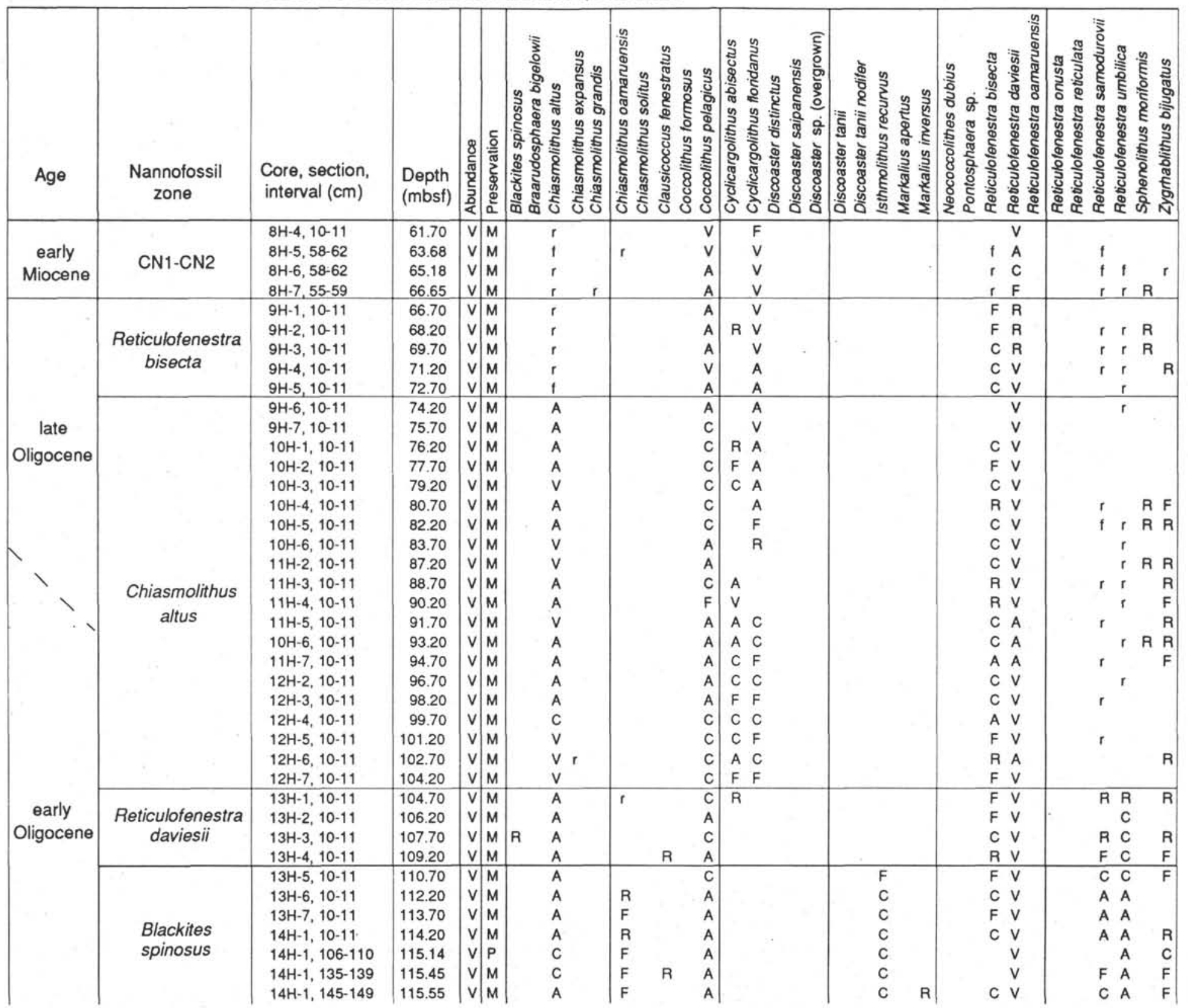

Notes: The high-latitude zonation of Wei and Wise (1990b) is used with the erection of the LO of Reticulofenestra bisecta as the marker for the Oligocene/Miocene boundary. Abundance is characterized by $V=$ very abundant, $A=$ abundant, $C=$ common, $F=$ few, $R=$ rare, $r=$ rare reworked specimens, and $f=$ few reworked specimens. For preservation, $\mathrm{M}=$ moderate and $\mathrm{G}=$ good. 
Table 3 (continued).

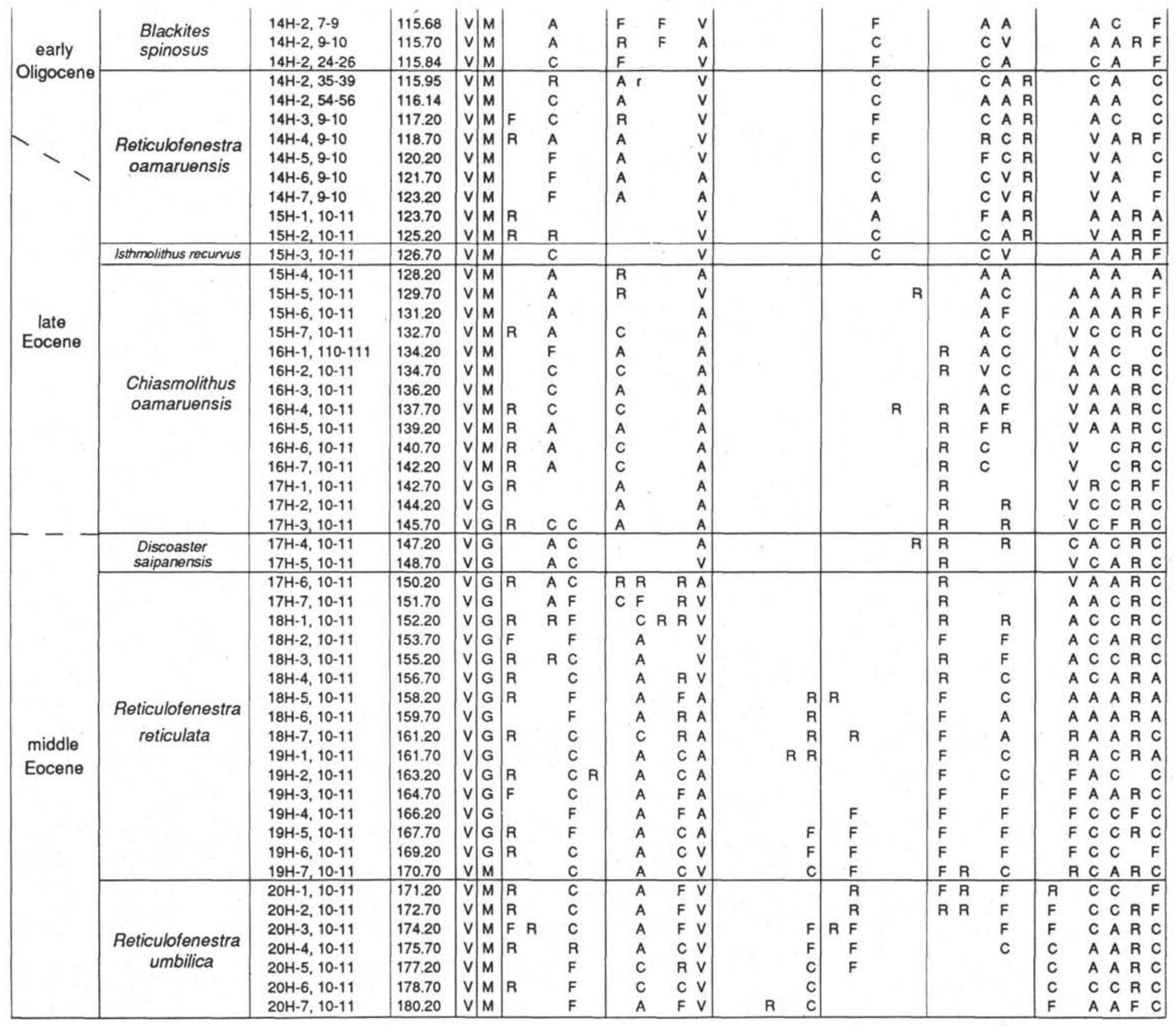




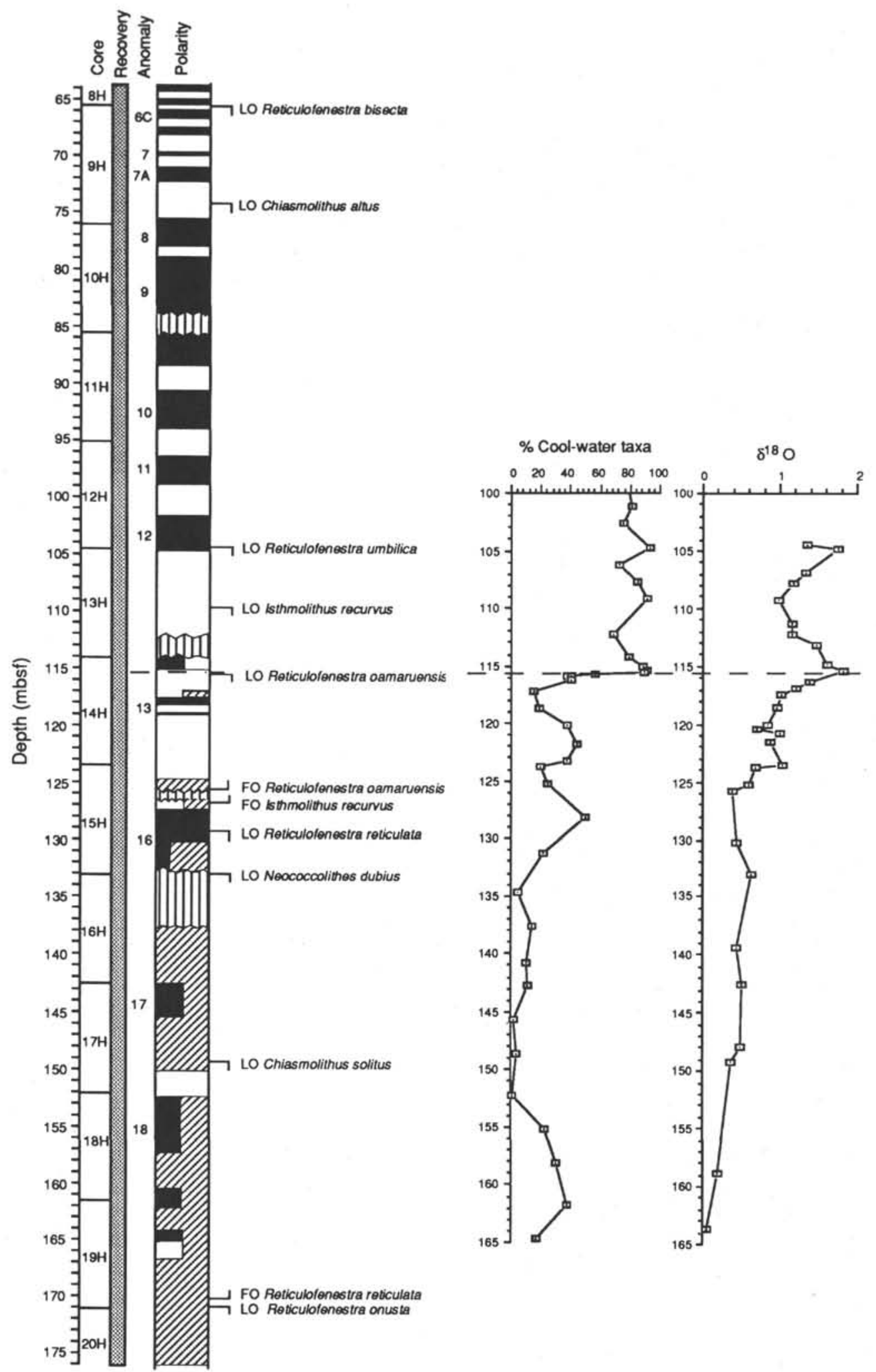

Figure 4. Calcareous nannofossil datum levels at Site 748. The magnetostratigraphy of Schlich, Wise, et al. (1989) is also shown. Magnetic normals are indicted by black, data gaps are shown by vertical lines, and data that has not been interpreted by diagonal lines. Polarity chrons based on less reliable data are indicated by one half the column. Changes in abundances of cool-water taxa (chiasmoliths + Isthmolithus recurvus + Reticulofenestra daviesii) and $\delta^{18} \mathrm{O}$ values of planktonic foraminifer Chiloguembelina cubensis (Zachos et al., this volume) are plotted on the right panel of the figure. The dashed line indicates the stratigraphic level where there is a coeval abrupt increase in the abundance of cool-water taxa and a large positive shift in the $\delta^{18} \mathrm{O}$ value of planktonic foraminifers (Zachos et al., this volume). 
Table 4. Stratigraphic levels of calcareous nannofossil datums and estimated ages, Site 748.

\begin{tabular}{lccc}
\hline \multicolumn{1}{c}{ Datum } & Depth (mbsf) & $\begin{array}{l}\text { Age } \\
\text { (Ma) }\end{array}$ & Source \\
\hline LO Reticulofenestra bisecta & $66.65-66.7$ & 24.0 & 1 \\
LO Chiasmolithus altus & $72.7-74.2$ & 27.0 & 2 \\
LO Reticulofenestra umbilica & $104.2-104.7$ & 33.0 & 2 \\
LO Isthmolithus recurvus & $109.2-110.7$ & 34.6 & 3 \\
LO Reticulofenestra oamaruensis & $115.84-115.95$ & 36.0 & 2 \\
FO Reticulofenestra oamaruensis & $125.2-126.7$ & 38.5 & 2 \\
FO Isthmolithus recurvus & $126.7-128.2$ & 39.0 & 2 \\
LO Chiasmolithus solitus & $148.7-150.2$ & 41.3 & 3 \\
\hline
\end{tabular}

Note: Data given are based on previous studies, as follows: $1=$ Wei and Wise (1989), 2 = Wei and Thierstein (1991), and 3 = Wei and Wise (1990b).

$43 \mathrm{Ma}$ (Wei and Wise, 1990a). This suggests that mid-latitude waters provided more favorable conditions for $R$. bisecta, and the species can be referred to as a temperate-water species.

It is also interesting to note that Reticulofenestra reticulata is about twice as abundant at high latitude Site 748 than at equatorial Site 711. A similar phenomenon has been observed in the South Atlantic where high-latitude Site 689 yielded twice as many $R$. reticulata as the mid-latitude sites (e.g., DSDP Sites 360, 516, and 523; Wei and Wise, 1990a). This contradicts the conclusions of Bukry (1977) and Aubry (1983), who classified $R$. reticulata as a warm-water species.

Based on the distribution patterns of calcareous nannofossil taxa in the South Atlantic latitudinal transect and on the results of a cluster analysis, Wei and Wise (1990a) were able to group the nannofossil taxa into warm-, temperate-, and cool-water taxa. They found it more useful to observe the abundance changes through time of warm- and cool-water taxa groups than those of individual species, which usually yield less robust interpretations because of floral evolution and small errors in the census data. Following this method, we present the abundance changes of warm-water taxa at Site 711 and cool-water taxa at Site 748 in Figures 12 and 13, respectively. The abundances of cool-water taxa at Site 711 and warm-water taxa at Site $\mathbf{7 4 8}$ are very low (generally less than a few percent), and their changes are not considered statistically significant.

Figure 12 indicates that there is a trend of decreasing abundance of warm-water taxa from the middle Eocene through the early Oligocene at Site 711. The abundance of warm-water taxa increased from $\sim 30 \%$ in the early Oligocene to $\sim 40 \%$ in the late Oligocene. It is interesting to note that the pattern of abundance change is quite similar to that of species diversity change at the site, with a general decrease from the middle Eocene to early Oligocene and a slight recovery in the late Oligocene.

The most distinct feature in Figure 13 is that there is an abrupt increase in the abundance of cool-water taxa (from $\sim 20 \%$ to over $90 \%$ ) at Site 748 within less than 0.5 m.y., from 36.3 $\mathrm{Ma}$ to $35.9 \mathrm{Ma}$. Coincident with this event is a large positive shift in the $\delta^{18} \mathrm{O}$ value of planktonic foraminifers, from $\sim 0.9 \%$ o to nearly $1.9 \%$ (Fig. 13 ). This sharp increase in cool-water taxa coeval with a large shift in the ${ }^{18} \mathrm{O}$ value of foraminifers has previously been recorded at Site 689 (Wei and Wise, 1990a, in press a; Stott et al., 1990; Fig. 14), a high-latitude site in the South Atlantic sector of the Southern Ocean $\left(65^{\circ} \mathrm{S}\right.$ latitude). The abundance of cool-water taxa remained relatively high for the rest of the Oligocene at both Site 748 (Fig. 13) and Site 689 (Fig. 14).

\section{DISCUSSION}

The discovery of an iridium anomaly at the Cretaceous/ Tertiary boundary in a number of sections led Alvarez et al. (1980) to suggest that the Cretaceous/Tertiary mass extinc-

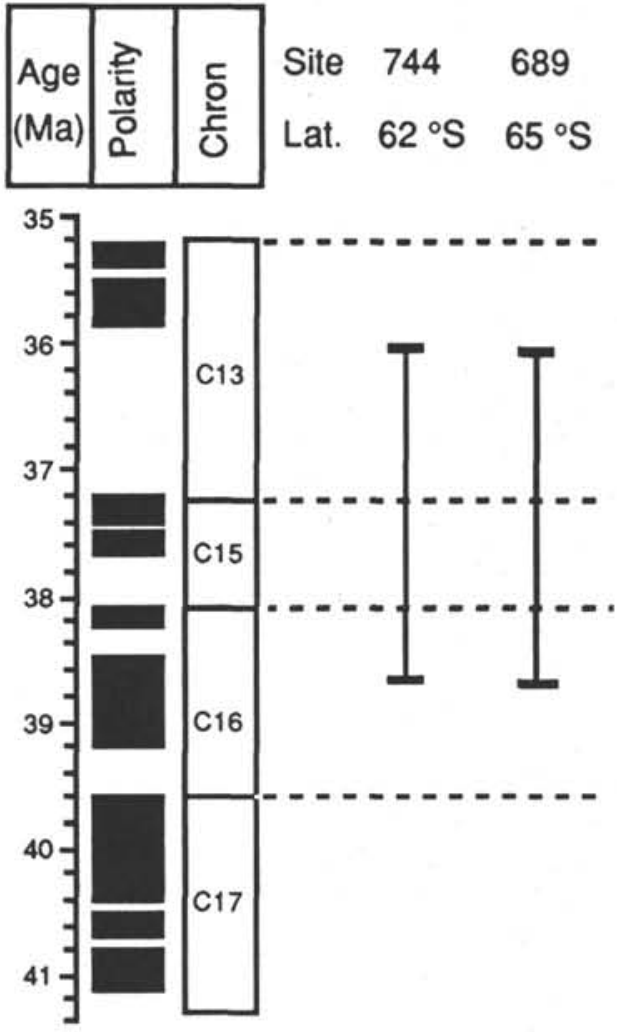

Figure 5. Magnetobiostratigraphic correlations of the FO and LO of Reticulofenestra oamaruensis at Sites 689 and 744 (Wei and Wise, in press b).

tions were the result of an extraterrestrial impact. Similarly, the simultaneous deposition of the North American tektites field and the extinction of five radiolarian species at the Eocene/Oligocene boundary (Glass and Zwart, 1979) led some authors (e.g., Alvarez et al., 1982; Asaro et al., 1982; Ganapathy, 1982) to suggest that a bolide event caused mass extinctions at the Eocene/Oligocene boundary. The EoceneOligocene extinctions have been prominently figured in the discussions of extinction periodicity, first proposed by Fischer and Arthur (1977) and Raup and Sepkoski (1984). The misconception was fostered by an overemphasis of the Terminal Eocene Event, which was the subject of IGCP Project 174 (Pomerol and Premoli-Silva, 1986).

Calcareous nannofossil data from Sites 711 and 748 show no mass extinctions at or near the Eocene/Oligocene boundary, in agreement with the results of Corliss et al. (1984). Instead, our data show that there is an abrupt increase (within $0.5 \mathrm{~m} . \mathrm{y}$.) in the abundance of cool-water taxa shortly after the Eocene/Oligocene boundary at Site 748 , indicating a sharp cooling of the surface waters in the high latitudes. The drop in surface-water temperature at Site 748 must be larger than $4^{\circ} \mathrm{C}$ because the cool-water nannofossil population change from $\sim 20 \%$ to $>90 \%$ is the same magnitude as that from $\sim 30^{\circ}$ to $65^{\circ} \mathrm{S}$ latitude in the late Eocene South Atlantic (Wei and Wise, 1990a), and the surface-water temperature difference between these latitudes at that time was at least $4^{\circ} \mathrm{C}$ (Shackleton and Boersma, 1981). This has important implications for the ice-volume change on Antarctica, as discussed below.

Zachos et al. (this volume) recorded about $1.0 \%$ increase in the $\delta^{18} \mathrm{O}$ values of planktonic foraminifers slightly above the Eocene/Oligocene boundary, at the same level that an abrupt increase in the abundance of cool-water taxa was found (Fig. 


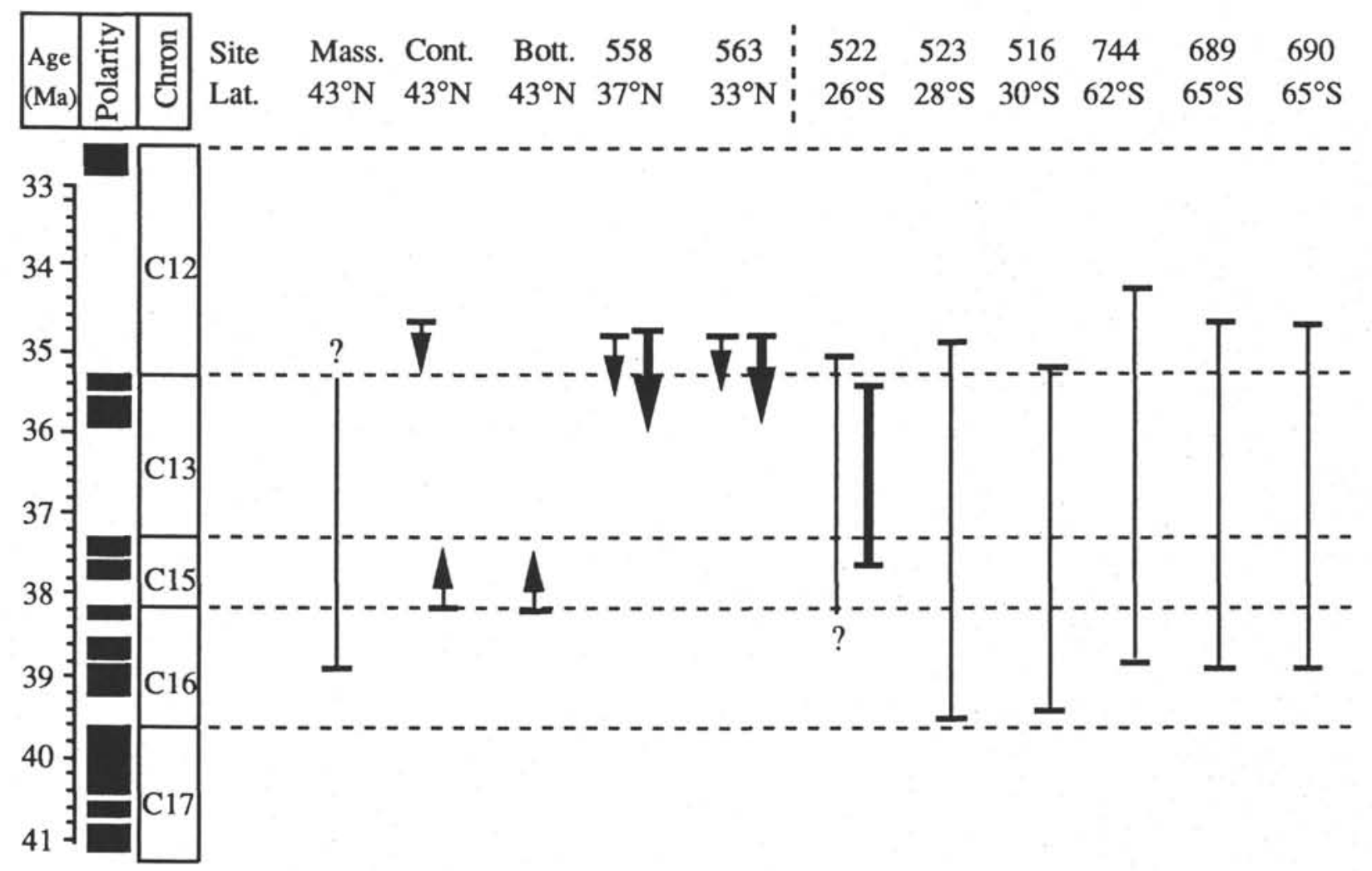

Figure 6. Correlation of the stratigraphic range of Isthmolithus recurvus with magnetostratigraphy at mid- and high-latitude sites (from Wei, this volume, Chapter 64). Data are taken from Coccioni et al. (1988), Mass. (Massignano, Italy); Monechi and Thierstein (1985), FO at Cont. (Contessa, Italy), Bott. (Bottacione, Italy); Lowrie et al. (1982), LO at Cont. (Contessa, Italy); Miller et al. (1985), DSDP Sites 558 and 563 (thinner lines); Parker et al. (1985), DSDP Sites 558 and 563 (thicker lines); Backman (1987), DSDP Site 522 (thinner line); Poore et al. (1984), DSDP Site 522 (thicker line), DSDP Site 523; Wei and Wise (1989), DSDP Site 516; Wei and Thierstein (1991), ODP Site 744; Wei and Wise (1990b), ODP Sites 689 and 690.

13). They interpreted that most of the $\delta^{18} \mathrm{O}$ value increase was caused by increased global ice volume and only about $0.2 \%$ $0.3 \%$ increase was due to a drop $\left(\sim 1^{\circ} \mathrm{C}\right)$ in surface-water temperature at the site. This was based on the observation of a coeval occurrence of ice-rafted debris (Breza and Wise, this volume) and the $\delta^{18} \mathrm{O}$ shift plus their compiled latitudinal $\delta^{18} \mathrm{O}$ gradient data (Zachos et al., this volume). These authors observed from their regression curves that there is only little change in the latitudinal $\delta^{18} \mathrm{O}$ gradient between the late Eocene and early Oligocene (only $0.2 \% 0-0.3 \%$ increase at $70^{\circ} \mathrm{S}$ latitude). This would suggest that surface waters in the high latitude cooled about $1^{\circ} \mathrm{C}$ from the late Eocene to the early Oligocene; otherwise, the latitudinal $\delta^{18} \mathrm{O}$ gradient would have been steeper from the late Eocene to the early Oligocene because surface-water temperatures in the tropics are supposed to remain fairly stable. This interpretation is rooted in a previous study by Keigwin and Corliss (1986).

There are several uncertainties in this interpretation:

1. The data Keigwin and Corliss (1986) and Zachos et al. (this volume) used to construct the latitudinal $\delta^{18} \mathrm{O}$ gradients are rough averages of late Eocene and early Oligocene $\delta^{18} \mathrm{O}$ values for planktonic foraminifers, and they are dependent on the density and distribution of the data used in the calculation. For example, if more data points from a $39-40 \mathrm{Ma}$ interval are used than from a $37-38 \mathrm{Ma}$ interval, the calculated result of the late Eocene $\delta^{18} \mathrm{O}$ values could be significantly different from one that uses more data from the $37-38 \mathrm{Ma}$ interval than from the 39-40 Ma interval.
2. The latitudinal $\delta^{18} \mathrm{O}$ gradients for the late Eocene and early Oligocene represented by linear regression lines (Keigwin and Corliss, 1986) or polynomial regression curves (Zachos et al., this volume) also depend on the density and distribution of the data points chosen along different latitudes. With only a few data points in the high latitudes (Keigwin and Corliss, 1986; Zachos et al., this volume), the regression lines (curves) were largely determined by the low- and mid-latitude data points.

3. The $R^{2}$ values for the regression lines (curves) are 0.65 or lower (Keigwin and Corliss, 1986; Zachos et al., this volume), indicating a rather poor fit of the data in the regression lines (curves). The latitudinal gradients obtained from those data sets show only general trends, which have large uncertainties in terms of value when considered statistically.

4 At best, the regression lines (curves) can depict the latitudinal $\delta^{18} \mathrm{O}$ gradients of the surface waters, but should not be viewed directly as latitudinal thermal gradients. For instance, the latitudinal $\delta^{18} \mathrm{O}$ gradient for the Holocene was shown to be $\sim 3.4 \%$ between $0^{\circ}$ and $60^{\circ}$ latitude (Keigwin and Corliss, 1986), which would give an apparent temperature difference of only $\sim 14^{\circ} \mathrm{C}$ between the equator and $60^{\circ}$ latitude. This direct interpretation is apparently wrong because the real temperature difference is about $28^{\circ} \mathrm{C}$. Other factors, such as salinity variations in the surface-waters at different latitudes, complicate the interpretation of the $\delta^{18} \mathrm{O}$ values in terms of surface-water temperatures (see discussion in Wei and Wise, 1990a).

Based on the above discussion, it is clear that one must view with caution the hypothesis that the latitudinal thermal 


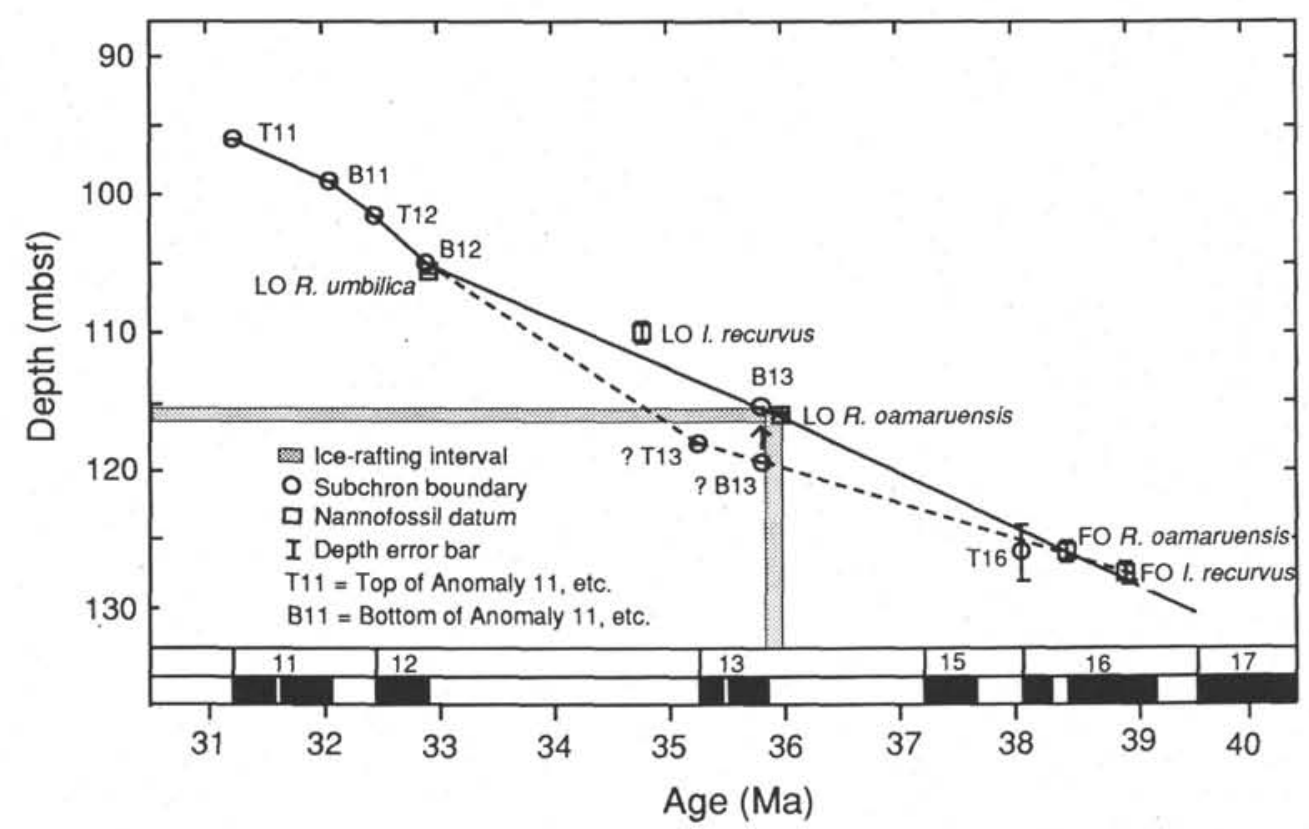

Figure 7. Age-depth curve for the upper Eocene-lower Oligocene interval at Site 748 based on magnetostratigraphy and calcareous nannofossil datums. Dashed lines are based on the magnetostratigraphic interpretation of Schlich, Wise, et al. (1989). Solid lines indicate interpretation in this paper. Note that moving the bottom of magnetic Subchron C13N from 119 to 115 mbsf, as indicated by the arrow, results in an age-depth curve that fits the nannofossil datums quite well. The Oligocene ice-rafted debris interval is dated as $35.8-36.0 \mathrm{Ma}$, an identical age for the Oligocene ice-rafted debris interval at Site 744 in the southern Kerguelen Plateau (see figure 4 of Wei, this volume, Chapter 64).

gradients remained virtually the same from late Eocene to early Oligocene times and that there was little further cooling in the high-latitude surface waters throughout this time. Calcareous nannofossil data from Site 748 suggest that there was a profound cooling in the high-latitude surface waters shortly after the Eocene/Oligocene boundary, and this sharp cooling was the most drastic and severe event during the EoceneOligocene interval. The latitudinal thermal gradient increased considerably at this time. Calcareous nannofossil data from Site 689 on Maud Rise agree with this result (Fig. 14). Moreover, coeval with the abrupt increase in cool-water taxa at Site 689 was a major and rapid change in the composition of clays derived from East Antarctica (Kennett and Barker, 1990). The smectite-dominated assemblages, typical products of chemical weathering under warm continental conditions, were replaced by clays dominated by illite and chlorite, typical products of physical weathering of parent rocks. This also indicates a drastic cooling on Antarctica near the Eocene/ Oligocene boundary.

If our estimate of the surface-water temperature drop of $4^{\circ} \mathrm{C}$ or more in the Eocene/Oligocene boundary transition at Site 748 is correct, the temperature drop would account for at least a $0.9 \%$ increase in the $\delta^{18} \mathrm{O}$ values of planktonic foraminifers as recorded by Zachos et al. (this volume). The remaining $0.1 \%$ or less increase in the $\delta^{18} \mathrm{O}$ value can then be attributed to the increase of ice volume in Antarctica. This estimated $0.1 \%$ change in isotope composition caused by an increase in ice volume is compatible with data from the tropics, such as that from DSDP Site 292, where planktonic foraminifers show a small increase $(0.2 \%)$ in $\delta^{18} \mathrm{O}$ values (Keigwin and Corliss, 1986, table 2). Significant warming of the tropical surface waters at this time, which would reduce $\delta^{18} \mathrm{O}$ values, has not been supported by any studies, including the present one. Planktonic foraminifers from the mid lati- tudes, such as at DSDP Sites 363,522 , and 540, show about a $0.5 \%$ increase in $\delta^{18} \mathrm{O}$ values across the Eocene/Oligocene boundary transition (Vergnaud Grazzini and Oberhänsli, 1986). A temperature decrease of $1^{\circ}-2^{\circ} \mathrm{C}$ across the Eocene/ Oligocene boundary transition in the mid-latitude surface waters is most likely, judging from the population change of planktonic fossils (Keller, 1983b; Wei and Wise, 1990a). This temperature drop would account for an $\sim 0.4 \%$ increase in the $\delta^{18} \mathrm{O}$ values. The remaining $\sim 0.1 \%$ can then be attributed to an isotope composition change in the sea waters caused by the increased ice volume on Antarctica. The latitudinal decrease in the amplitude of the $\delta^{18} \mathrm{O}$ shift recorded in planktonic foraminifers, with the largest in the high latitudes and the smallest in the low latitudes, has previously been noted by a number of investigators (e.g., Keigwin, 1980; Keigwin and Corliss, 1986; Vergnaud Grazzini and Oberhänsli, 1986). This phenomenon is a strong indication that the $\delta^{18} \mathrm{O}$ shift is primarily a temperature drop in the high and mid latitudes, with a minor proportion attributed to the isotope composition change in sea waters caused by an increased ice volume on Antarctica. If we assume that the average $\delta^{18} \mathrm{O}$ value for the ice on Antarctica in the Paleogene was about $-30 \%$, or $20 \%$ o heavier than that of the present Antarctic ice sheet (Shackleton and Kennett, 1975), and take $1380 \times 10^{6} \mathrm{~km}^{3}$ as the volume of the oceans during the Paleogene, an increase of $0.1 \%$ in the $\delta^{18} \mathrm{O}$ value of the world's oceans means that the ice volume on Antarctica increased about $4.6 \times 10^{3} \mathrm{~km}^{3}(\sim 20 \%$ of the present Antarctic ice sheet).

Because abundant ice-rafted debris has been found in the lowest Oligocene at ODP Sites 738, 744 (Ehrmann, 1991), and 748 (Breza and Wise, this volume), and extensive lowermost Oligocene glaciomarine sediment has been recovered in the Ross Sea (Barrett et al., 1989) and in Prydz Bay, East Antarctica (Barron, Larsen, et al., 1989), it has been inferred 
W. WEI, G. VILLA, S. W. WISE, JR.

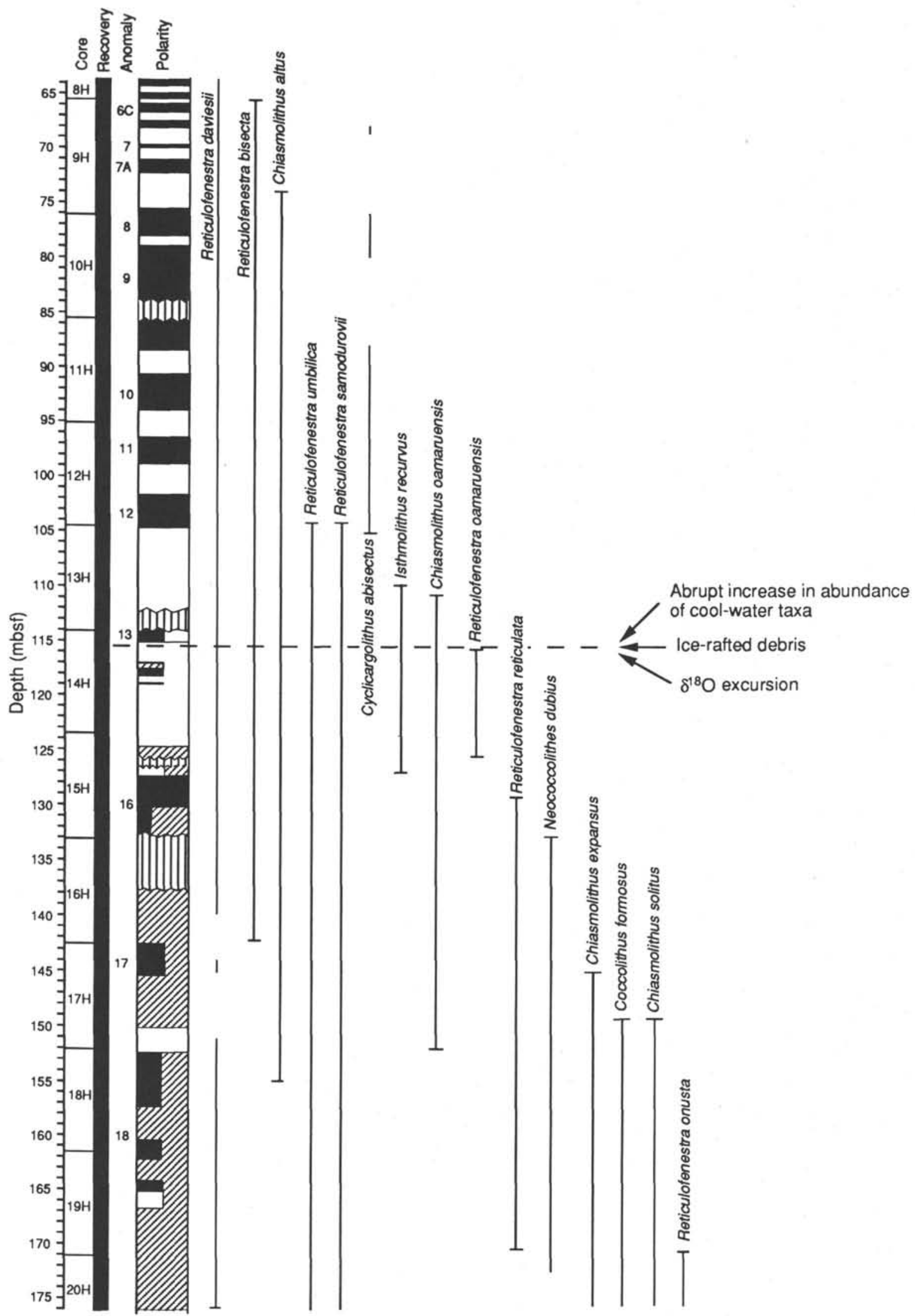

Figure 8. Reinterpreted magnetostratigraphy of Site 748 and its correlation with the calcareous nannofossil datums. The dashed line indicates the stratigraphic level where there is a coeval abrupt increase in the abundance of cool-water taxa, a large positive shift in the $\delta^{18} \mathrm{O}$ value of planktonic foraminifers (Zachos et al., this volume), and the occurrence of ice-rafted debris (Breza and Wise, this volume). 


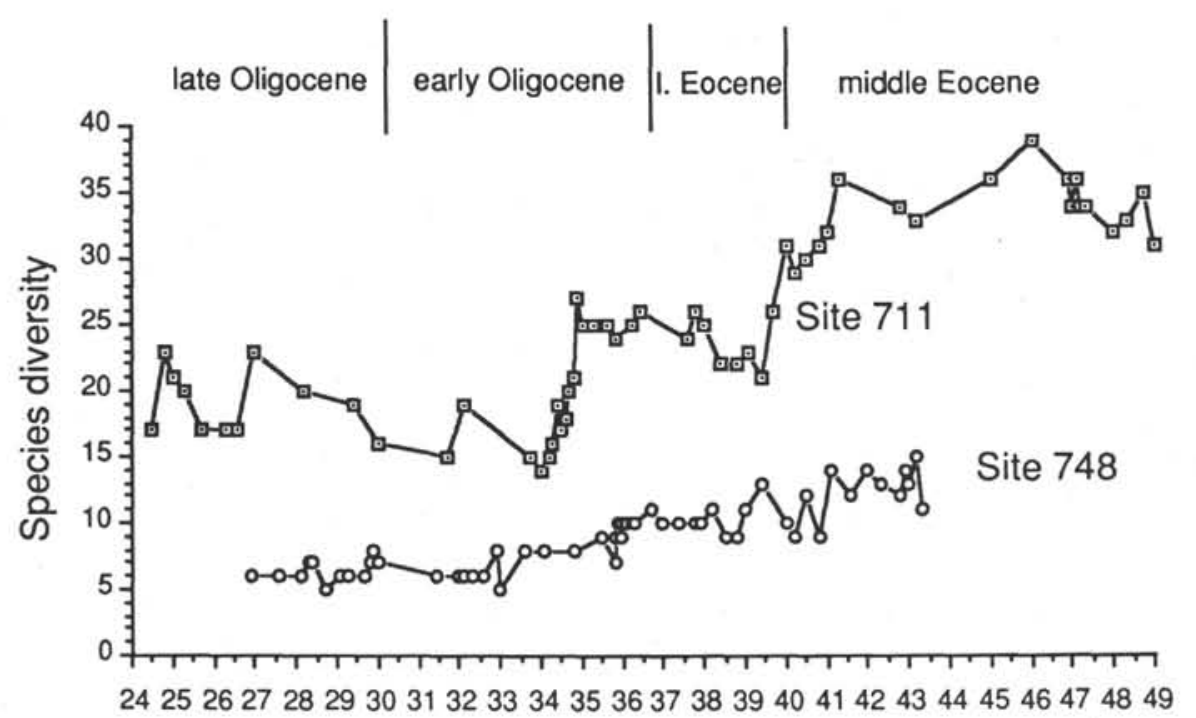

Age (Ma)

Figure 9. Calcareous nannofossil species diversity change through time, Sites 711 and 748.

that the Antarctic ice sheet extended further seaward (at least in some areas) in the earliest Oligocene than today (Barron et al., 1991; Wise et al., 1991, and this volume; Wei, this volume, Chapter 64). In terms of volume, the ice-sheet model of Robin (1988) suggests that the ice sheet in the earliest Oligocene reached virtually the same size as the present one. If we tentatively take the volume of the earliest Oligocene ice sheet as only half the size of the present one, an ice sheet $40 \%$ the size of the present one could exist in the latest Eocene based on our interpretation of the nannofossil and $\delta^{18} \mathrm{O}$ data as discussed above. We note that this interpretation is not incompatible with the findings in Prydz Bay, where long sequences of glaciomarine sediment below the lower Oligocene glaciomarine sediment indicate extensive glacial activities on Antarctica in the earliest Oligocene and possibly as early as the middle Eocene (Barron et al., 1991). In fact, significant ice on Antarctica in the early and middle Eocene is also a possibility, as discussed by Wei (this volume, Chapter 63).

\section{CONCLUSIONS}

Eocene-Oligocene calcareous nannofossil biostratigraphy and the quantitative nannofossil data for Site 748 have enabled us to reinterpret the preliminary magnetostratigraphy. Calcareous nannofossil species diversity is low at Site 748 relative to lower latitude sites, with about 13 taxa in the middle Eocene and decreasing to about 6 in the late Oligocene. There is, however, no apparent mass extinction at any stratigraphic level, including the Eocene/Oligocene boundary. Similarly, no mass extinctions were recorded at or near the Eocene/Oligocene boundary at Site 711. Species diversity at equatorial Site 711 is significantly higher, with a maximum of 39 species in the middle Eocene and a minimum of 14 species in the early Oligocene. The abundance patterns of nannofossil taxa are also quite different at the two sites. Chiasmoliths, Isthmolithus recurvus, and Reticulofenestra daviesii are abundant at high-latitude Site 748 , but their abundances are greatly reduced or are virtually absent at equatorial Site 711. On the other hand, limited Coccolithus formosus, discoasters, and sphenoliths were recorded only in the middle Eocene at Site 748 in contrast to their high abundances at Site 711. This indicates that there was a significant latitudinal biogeographic gradient between the equatorial region and the high latitudes of the Indian Ocean for the middle Eocene-Oligocene interval.

The abundance change in warm-water taxa is similar to the change in species diversity at Site 711. There is a general trend of decreasing abundance of warm-water taxa from the middle Eocene through the early Oligocene at Site 711, suggesting a gradual cooling of the surface waters in the equatorial Indian Ocean. The abundance of warm-water taxa increased in the late Oligocene, in association with an increase in species diversity, and this increase may reflect a warming of the surface waters in the late Oligocene.

The most distinct feature at high-latitude Site 748 is an abrupt increase in the abundance of cool-water taxa (from $\sim 20 \%$ to over $90 \%$ ) in less than $0.5 \mathrm{~m} . \mathrm{y}$., from $36.3 \mathrm{Ma}$ to $35.9 \mathrm{Ma}$. Coincident with this event was an $\sim 1.0 \%$ positive shift in $\delta^{18} \mathrm{O}$ values of planktonic foraminifers. This sharp increase in cool-water taxa coeval with a large positive shift in $\delta^{18} \mathrm{O}$ values has previously been recorded at the bottom of magnetic Subchron C13N ( $\sim 35.9 \mathrm{Ma})$ at ODP Site 689 in the Weddell Sea and is believed to be a useful biostratigraphic event for locating the bottom of Subchron $\mathrm{C} 13 \mathrm{~N}$ in the Southern Ocean. The abrupt increase in cool-water taxa coeval with a large positive shift in $\delta^{18} \mathrm{O}$ values of planktonic foraminifers suggests that the high-latitude surface waters drastically cooled around 36.3-35.9 Ma. The temperature drop was estimated to be $>4^{\circ}$ at Site 748 (accounting for $>0.9 \%$ increase in $\delta^{18} \mathrm{O}$ values), based on the nannofossil population change at Site 748 relative to the latitudinal biogeographic gradient established in the South Atlantic Ocean (Wei and Wise, 1990a). The remaining $\sim 0.1 \%$ o increase in $\delta^{18} \mathrm{O}$ values can then be attributed to an ice-volume increase on Antarctica. Consequently, the $\sim 1.0 \%$ increase in the $\delta^{18} \mathrm{O}$ values of planktonic foraminifers at Site 748 is a result largely of the temperature drop in the high latitudes rather than an ice-volume signal. The $\sim 0.1 \%$ increase in $\delta^{18} \mathrm{O}$ values translates into an ice-volume increase of $4.6 \times$ $10^{3} \mathrm{~km}^{3}$, or $\sim 20 \%$ the size of the present Antarctic ice sheet. 


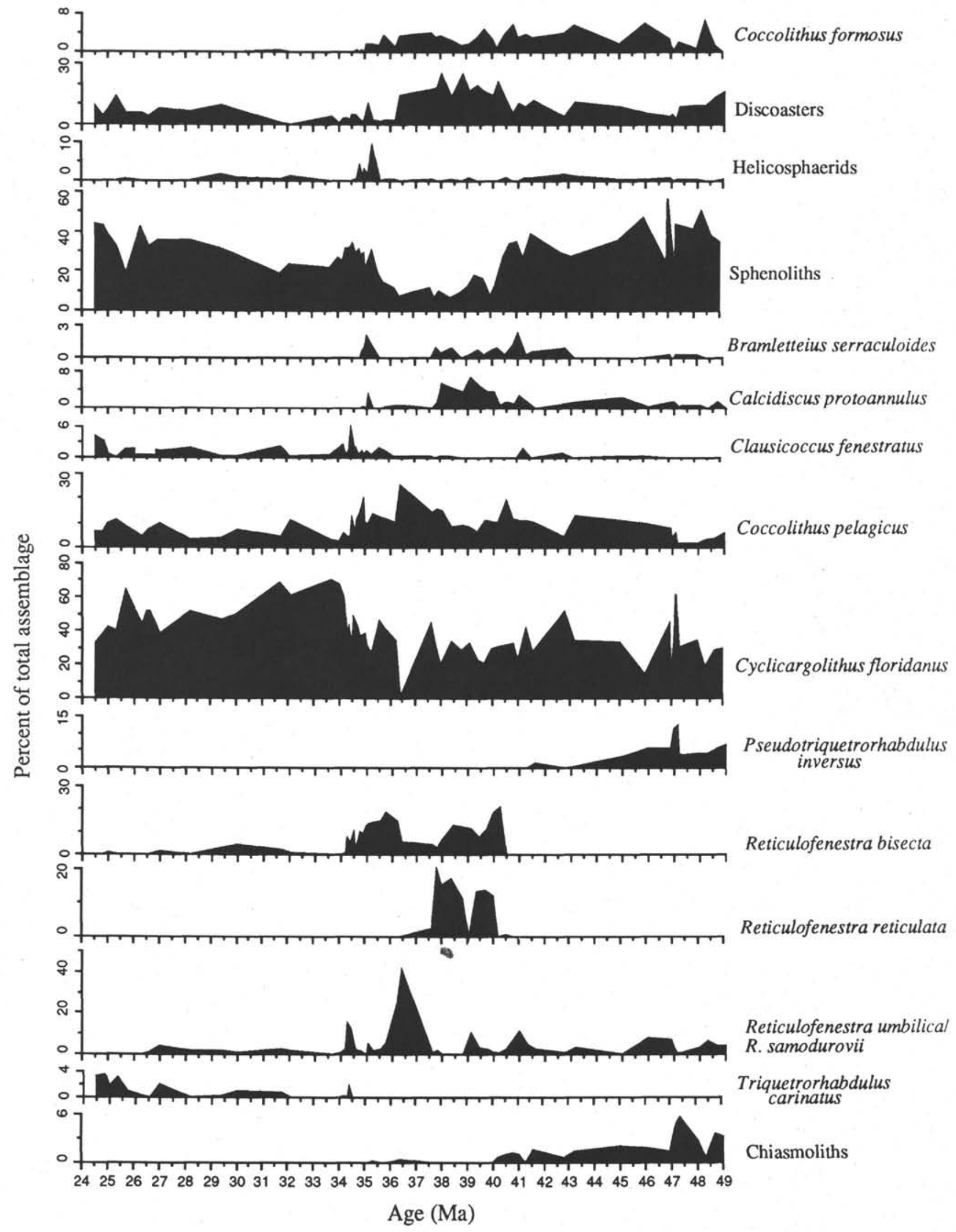

Figure 10. Abundance patterns of calcareous nannofossil taxa at Site 711. 


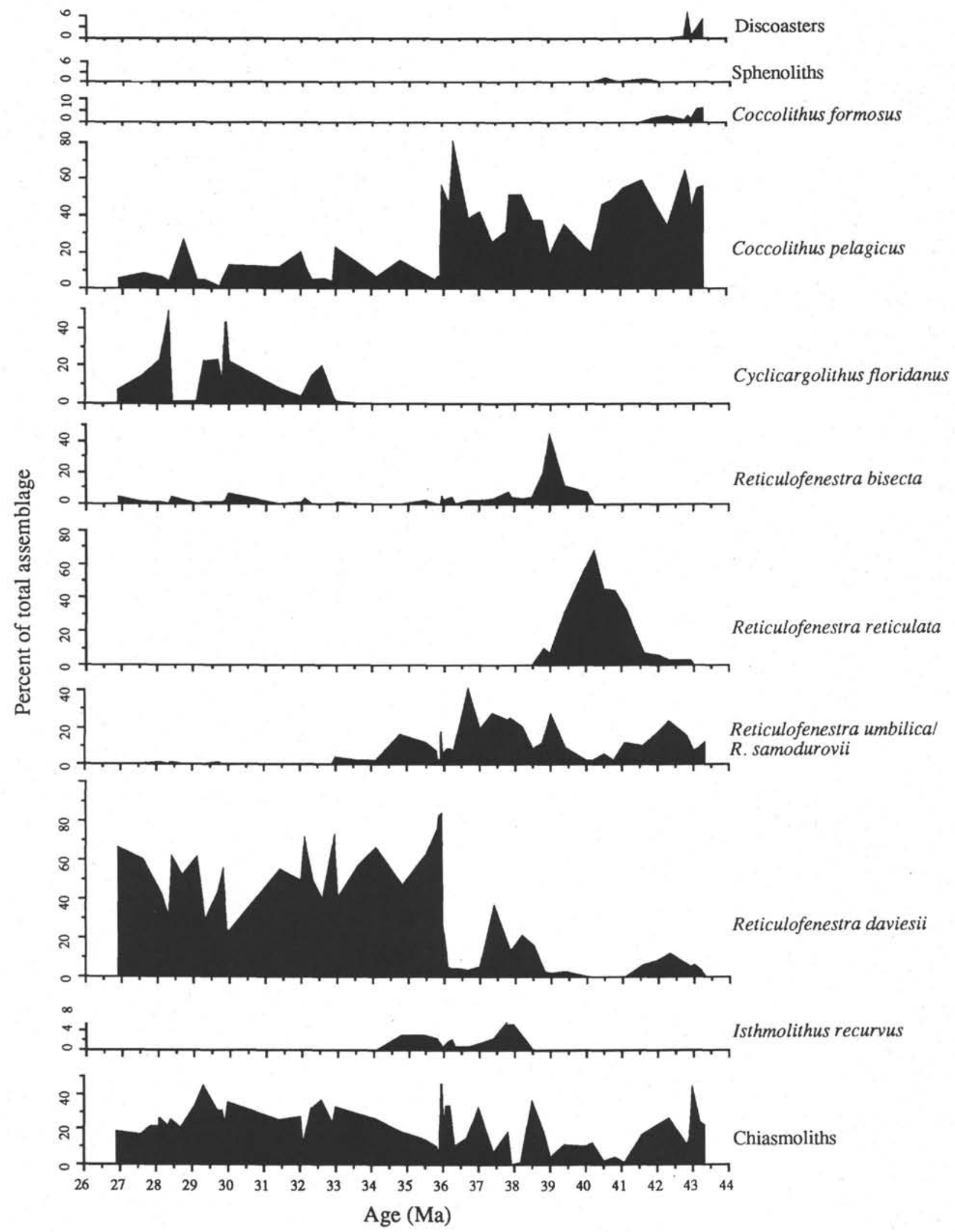

Figure 11. Abundance patterns of calcareous nannofossil taxa at Site 748. 
W. WEI, G. VILLA, S. W. WISE, JR.

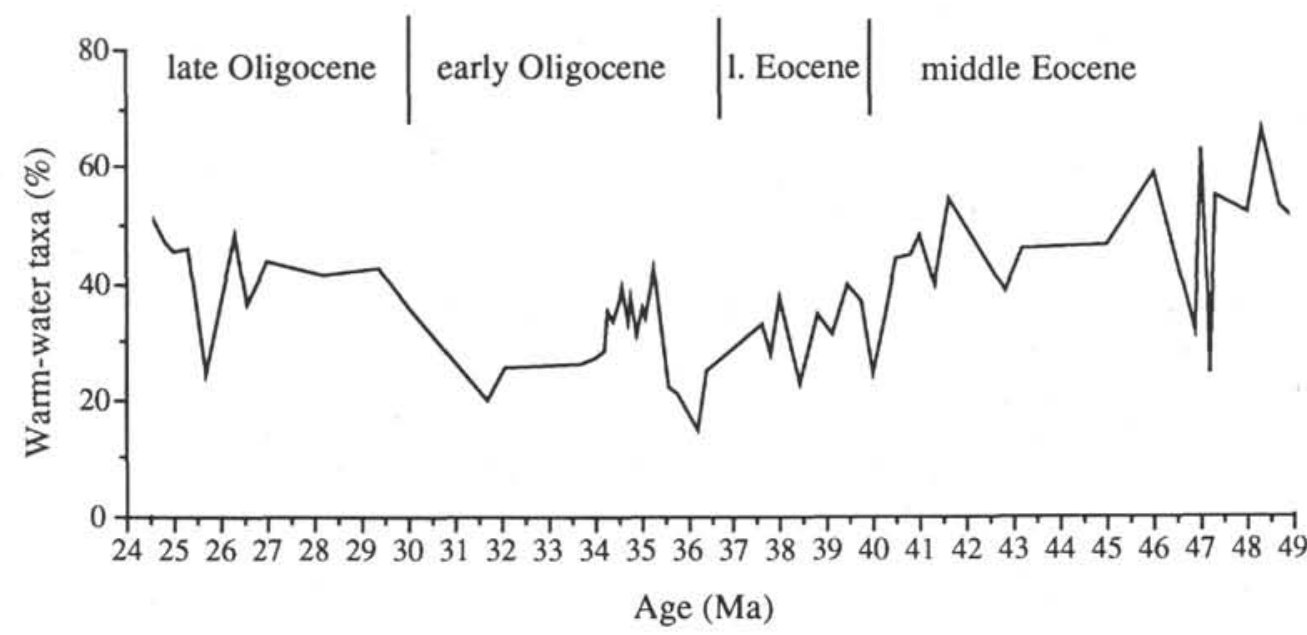

Figure 12. Abundance change of warm-water taxa (Coccolithus formosus + discoasters + helicosphaerids + sphenoliths) through time at Site 711 .

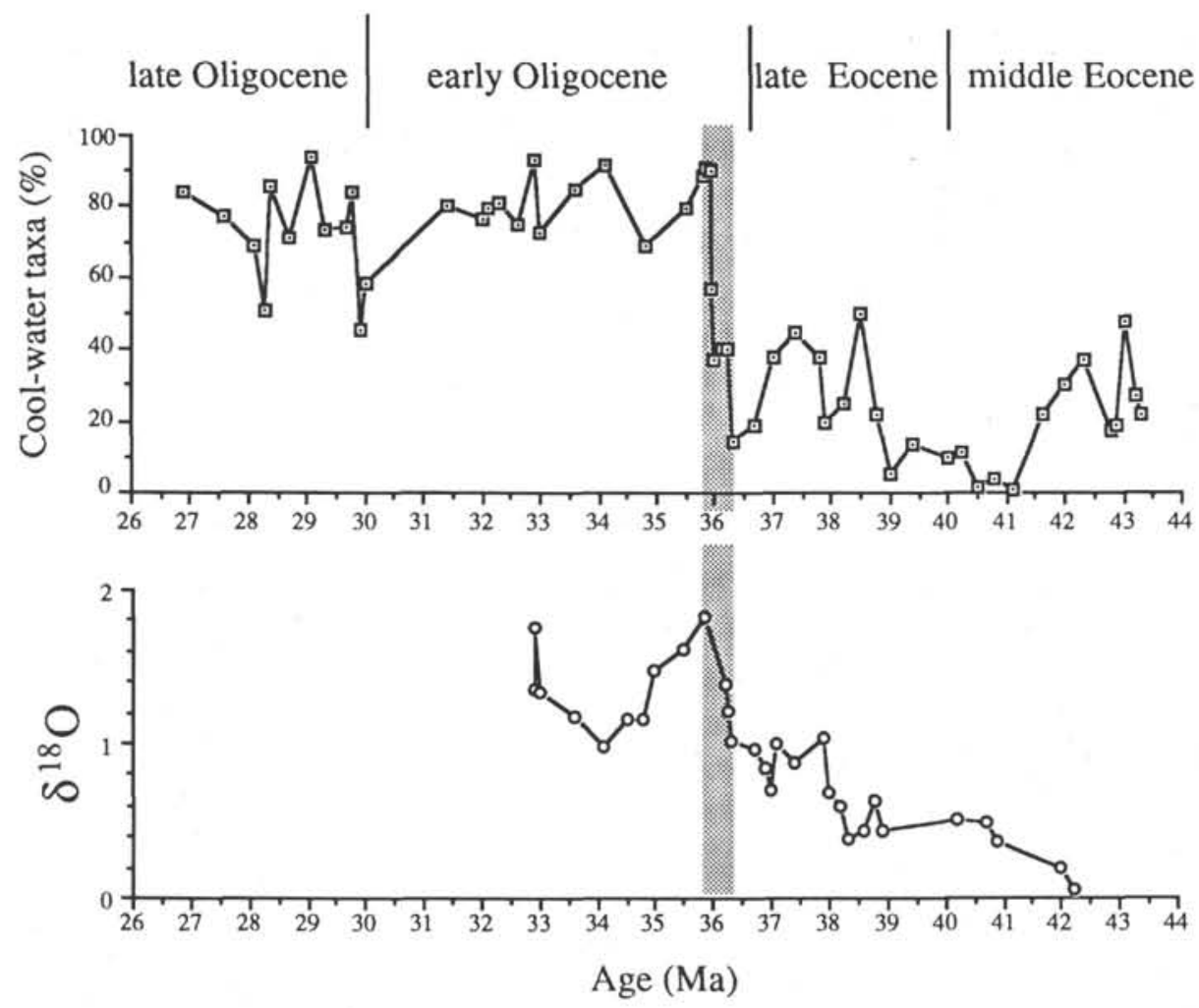

Figure 13. Abundance change of cool-water taxa (chiasmoliths + Isthmolithus recurvus + Reticulofenestra daviesii) through time at Site 748 . Changes in $\delta^{18} \mathrm{O}$ values of planktonic foraminifers (Chiloguembelina cubensis) through time at the site are shown in the lower panel. Isotope data are taken from Zachos et al. (this volume). Note the abrupt increase in cool-water taxa and the large positive shift in the $\delta^{18} \mathrm{O}$ value of planktonic foraminifers as indicated by the shaded bar.

\section{ACKNOWLEDGMENTS}

We wish to thank L. M. Bybell (U.S. Geological Survey) and $\mathrm{E}$. Joyce (Unocal) for critical reviews and helpful suggestions. D. Lazarus (Swiss Federal Institute of Technology) kindly provided the Age-Depth Plot program, which was used to construct Figure 7. Samples were provided by NSF through the Ocean Drilling Program. This study was supported by NSF grant No. DPP89-17976 and grants from the U.S. Science Advisory Committee.

\section{REFERENCES}

Alvarez, L. W., Alvarez, W., Asaro, F., and Michel, H. V., 1980. Extraterrestrial cause for the Cretaceous-Tertiary extinction. Science, 208:1095-1108. 
Alvarez, W., Asaro, F., Michel, H. V., and Alvarez, L. W., 1982. Iridium anomaly approximately synchronous with terminal Eocene extinctions. Science, 216:886-888.

Asaro, F., Alvarez, L. W., Alvarez, W., and Michel, H. V., 1982. Geochemical anomalies near the Eocene/Oligocene and Permian/ Triassic boundaries. In Silver, L. T., and Schultz, P. H. (Eds.), Geological Implications of Impacts of Large Asteroids and Comets on the Earth. Spec. Pap. Geol. Soc. Am., 190:517-528.

Aubry, M.-P., 1983. Late Eocene to early Oligocene calcareous nannoplankton biostratigraphy and biogeography. AAPG Bull., 67:415.

Backman, J., 1987. Quantitative calcareous nannofossil biochronology of middle Eocene through early Oligocene sediment from DSDP Sites 522 and 523. Abh. Geol. Bundesanst. Austria, 39:21-31.

Barrera, E., and Huber, B. T., 1991. Paleogene and early Neogene oceanography of the southern Indian Ocean: Leg 119, foraminifer stable isotope results. In Barron, J., Larsen, B., et al., Proc. ODP, Sci. Results, 119: College Station, TX (Ocean Drilling Program), 731-738.

Barrett, P. J., Hambrey, M. J., Harwood, D. M., Pyne, A. R., and Webb, P.-N., 1989. Synthesis. In Barrett, P. J. (Ed.), Antarctic Cenozoic History from the CIROS-1 Drillhole, McMurdo Sound. DSIR Bull. N. Z., 245:241-251.

Barron, J., Baldauf, J. G., Barrera, E., Caulet, J.-P., Huber, B. T., Keating, B. H., Lazarus, D., Sakai, H., Thierstein, H. R., and Wei, W., 1991. Biochronologic and magnetochronologic synthesis of ODP Leg 119 sediments from the Kerguelen Plateau and Prydz Bay, Antarctica. In Barron, J., Larsen, B., et al., Proc. ODP, Sci. Results, 119: College Station, TX (Ocean Drilling Program), 813-848.

Barron, J., Larsen, B., and Baldauf, J. G., 1991. Evidence for late Eocene-early Oligocene Antarctic glaciation and observations of late Neogene glacial history of Antarctica: results from ODP Leg 119. In Barron, J., Larsen, B., et al., Proc. ODP, Sci. Results, 119: College Station, TX (Ocean Drilling Program), 869-894.

Barron, J., Larsen, B., et al., 1989. Proc. ODP, Init. Repts., 119: College Station, TX (Ocean Drilling Program).

Benson, R. H., 1975. The origin of the psychrosphere as recorded in changes of deep-sea ostracode assemblages. Lethaia, 8:69-83.

Berger, W. H., 1973. Cenozoic sedimentation in the eastern tropical Pacific. Geol. Soc. Am. Bull., 84:1941-1954.

Berggren, W. A., Kent, D. V., and Flynn, J. J., 1985. Jurassic to Paleogene: Part 2. Paleogene geochronology and chronostratigraphy. In Snelling, N. J. (Ed.), The Chronology of the Geological Record. Geol. Soc. London Mem., 10:141-195.

Bukry, D., 1977. Cenozoic coccolith and silicoflagellate stratigraphy, offshore northwest Africa, Deep Sea Drilling Project Leg 41. In Lancelot, Y., Seibold, E., et al., Init. Repts. DSDP, 41: Washington (U.S. Govt. Printing Office), 689-719.

Coccioni, R., Monaco, P., Monechi, S., Nocchi, M., and Parisi, G., 1988. Biostratigraphy of the Eocene/Oligocene boundary at Massignano (Ancona, Italy). In Premoli Silva, I., Coccioni, R., and Montanari, A. (Eds.), The Eocene/Oligocene Boundary in the Marche-Umbria Basin (Italy): Ancona (Industrie Grafiche Filli Aniballi), 59-80.

Corliss, B. H., Aubry, M.-P., Berggren, W. A., Fenner, J. M., Keigwin, D., Jr., and Keller, G., 1984. The Eocene/Oligocene boundary event in the deep sea. Science, 226:806-810.

Ehrmann, W. U., 1991. Sediment composition on Southern Kerguelen Plateau and its implications for paleoclimate and depositional environment. In Barron, J., Larsen, B., et al., Proc. ODP, Sci. Results, 119: College Station, TX (Ocean Drilling Program), 185-210.

Fischer, A. G., and Arthur, M. A., 1977. Secular variations in the pelagic realm. In Cook, H. E., and Enos, P. (Eds.), Deep Water Carbonate Environments. Spec. Publ. Soc. Econ. Paleontol. Mineral., 25:19-50.

Ganapathy, R., 1982. Evidence for major meteorite impact on the earth 34 million years ago: implications for Eocene extinctions. Science, 216:885-886.

Geitzenauer, K. R., 1969. Coccoliths as late Quaternary paleoclimatic indicators in the subantarctic Pacific Ocean. Nature, 223:170-172.

Glass, B. P., and Zwart, M. J., 1979. North American microtektites in Deep Sea Drilling Project cores from the Caribbean Sea and Gulf of Mexico. Geol. Soc. Am. Bull., 90:595-602.
Haq, B. U., Hardenbol, J., and Vail, P. R., 1987. Chronology of fluctuating sea levels since the Triassic. Science, 235:1156-1167.

Haq, B. U., and Lohmann, G. P., 1976. Early Cenozoic calcareous nannoplankton biogeography of the Atlantic Ocean. Mar. Micropaleontol., 1:119-194.

Haq, B. U., Premoli-Silva, I., and Lohmann, G. P., 1977. Calcareous plankton paleobiogeographic evidence for major climatic fluctuations in the early Cenozoic Atlantic Ocean. J. Geophys. Res., 82:3861-3876.

Heath, G. R., 1969. Carbonate sedimentation in the abyssal equatorial Pacific during the past 50 million years. Geol. Soc. Am. Bull., 80:689-694.

Hess, J., Stott, L. D., Bender, M. L., Kennett, J. P., and Schilling, J.-G., 1990. The Oligocene marine microfossil record: age assessments using strontium isotopes. Paleoceanography, 4:655-679.

Keigwin, H. D., 1980. Palaeoceanographic change in the Pacific at the Eocene/Oligocene boundary. Nature, 287:722-725.

Keigwin, H. D., and Corliss, B. H., 1986. Stable isotopes in late middle Eocene to Oligocene foraminifera. Geol. Soc. Am. Bull., 97:335-345.

Keller, G., 1983a. Paleoclimatic analyses of middle Eocene through Oligocene planktic foraminiferal faunas. Palaeogeogr., Palaeoclimatol., Palaeoecol., 43:73-94.

1983b. Biochronology and paleoclimatic implications of middle Eocene to Oligocene planktic foraminiferal faunas. Mar. Micropaleontol., 7:463-486.

1986. Stepwise mass extinctions and impact events: late Eocene to early Oligocene. Mar. Micropaleontol., 10:267-293.

Keller, G., D'Hondt, S., and Vallier, T. L., 1983. Multiple microtectite horizons in upper Eocene marine sediments: no evidence for mass extinctions. Science, 221:150-152.

Kennett, J. P., 1977. Cenozoic evolution of Antarctic glaciation, the circum-Antarctic ocean, and their impact on global paleoceanography. J. Geophys. Res., 82:3843-3860.

1983. Paleoceanography: global ocean evolution. Rev. Geophys. Space Phys., 21:1258-1274.

Kennett, J. P., and Barker, P. F., 1990. Latest Cretaceous to Cenozoic Climate and oceanographic developments in the Weddell Sea, Antarctica: an ocean-drill perspective. In Barker, P. F., Kennett, J. P., Proc. ODP, Sci. Results., 113: College Station, TX (Ocean Drilling Program), 937-960.

Loeblich, A. R., and Tappan, H., 1966. Annotated index and bibliography of the calcareous nannoplankton. Phycologia, 5:81-215. 1968. Annotated index and bibliography of the calcareous nannoplankton II. J. Paleontol., 42:584-598.

,1969. Annotated index and bibliography of the calcareous nannoplankton III. J. Paleontol., 43:568-588.

1970a. Annotated index and bibliography of the calcareous nannoplankton IV. J. Paleontol., 44:558-574.

1970b. Annotated index and bibliography of the calcareous nannoplankton V. Phycologia, 9:157-174.

1971. Annotated index and bibliography of the calcareous nannoplankton VI. Phycologia, 10:315-339.

1973. Annotated index and bibliography of the calcareous nannoplankton VII. J. Paleontol., 47:715-759.

Lowrie, W., Alvarez, W., Napoleone, G., Perch-Nielsen, K., Premoli Silva, I., and Toumarkine, M., 1982. Paleogene magnetic stratigraphy in Umbrian pelagic carbonate rocks: the Contessa sections, Gubbio. Geol. Soc. Am. Bull., 93:411-432.

Matthews, R. K., and Poore, R. Z., 1980. Tertiary $\delta^{18} \mathrm{O}$ record and glacio-eustatic sea-level fluctuations. Geology, 8:501-504.

McIntyre, A., and Bé, A.W.H., 1967. Modern Coccolithophoridae of the Atlantic Ocean. I. placoliths and crytoliths. Deep-Sea Res. Oceanogr. Abstr., 14:561-597.

McIntyre, A., Bé, A.W.H., and Roche, M. B., 1970. Modern Pacific coccolithophorida: a paleontological thermometer. Trans. N. Y. Acad. Sci., 32:720-731.

Miller, K. G., Aubry, M.-P., Khan, M. J., Melillo, A. J., Kent, D. V., and Berggren, W. A., 1985. Oligocene-Miocene biostratigraphy, magnetostratigraphy, and isotopic stratigraphy of the western North Atlantic. Geology, 13:257-261.

Monechi, S. and Thierstein, H. R., 1985. Late Cretaceous-Eocene nannofossil and magnetostratigraphic correlations near Gubbio, Italy. Mar. Micropaleontol., 9:419-440. 
Oberhänsli, H., and Toumarkine, M., 1985. The Paleogene oxygen and carbon isotope history of Sites 522, 523, and 524 from the central South Atlantic. In Hsü, K. J., and Weissert, H. J. (Eds.), South Atlantic Paleoceanography: Oxford (Oxford Press), 124-147.

Okada, H., 1990. Quaternary and Paleogene calcareous nannofossils, Leg 115. In Duncan, R. A., Backman, J., Peterson, L. C., et al., Proc. ODP, Sci. Results, 115: College Station, TX (Ocean Drilling Program), 129-174.

Okada, H., and Bukry, D., 1980. Supplementary modification and introduction of code numbers to the low-latitude coccolith biostratigraphic zonation (Bukry 1973; 1975). Mar. Micropaleontol., $5: 321-325$.

Okada, H., and Honjo, S., 1973. The distribution of oceanic coccolithophorids in the Pacific. Deep-Sea Res. Oceanogr. Abstr., 20:355-374.

Parker, M. E., Clark, M., and Wise, S. W., Jr., 1985. Calcareous nannofossils of Deep Sea Drilling Project Sites 558 and 563, North Atlantic Ocean: biostratigraphy and the distribution of Oligocene braarudosphaerids. In Bougault, H., Cande, S. C., et al., Init. Repts. DSDP, 82: Washington (U.S. Govt. Printing Office), 559-589.

Pomerol, C., and Premoli-Silva, I., (Eds.), 1986. Terminal Eocene Events: Amsterdam (Elsevier).

Poore, R. Z., and Matthews, R. K., 1984a. Late Eocene-Oligocene oxygen and carbon isotope record from South Atlantic Ocean, Deep Sea Drilling Project Site 522. In Hsü, K. J., LaBrecque, J. L., et al., Init. Repts DSDP, 73: Washington (U.S. Govt. Printing Office), 725-735.

$1984 \mathrm{~b}$. Oxygen isotope ranking of late Eocene and Oligocene planktonic foraminifers: implications for Oligocene seasurface temperatures and global ice-volume. Mar. Micropaleontol., 9:111-134.

Poore, R. Z., Tauxe, L., Percival, S. F., Jr., LaBrecque, J. L., Wright, R., Petersen, N. P., Smith, C. C., Tucker, P., and Hsü, K. J., 1984. Late Cretaceous-Cenozoic magnetostratigraphic and biostratigraphic correlations for the South Atlantic Ocean, Deep Sea Drilling Project Leg 73. In Hsü, K. J., LaBrecque, J., et al., Init. Repts. DSDP, 73: Washington (U.S. Govt. Printing Office), 645-655.

Prentice, M. L., and Matthews, R. K., 1988. Cenozoic ice-volume history: development of a composite oxygen isotope record. Geology, 16:963-966.

Raup, D. M., and Sepkoski, J. J., Jr., 1984. Periodicity of extinctions in the geologic past. Proc. Nat. Acad. Sci. U.S.A., 81:801-805.

Robin, G. de Q., 1988. The Antarctic ice sheet, its history and response to sea level and climate changes over the past 100 million years. Palaeogeogr., Palaeoclimatol., Palaeoecol., 67:31-50.

Savin, S. M., Douglas, R. G., and Stehli, F. G., 1975. Tertiary marine paleotemperatures. Geol. Soc. Am. Bull., 86:1499-1510.

Schlich, R., Wise, S. W., Jr., et al., 1989. Proc. ODP, Init. Repts., 120: College Station, TX (Ocean Drilling Program).

Shackleton, N. J., and Boersma, A., 1981. The climate of the Eocene ocean. J. Geol. Soc. London, 138:153-157.

Shackleton, N. J., and Kennett, J. P., 1975. Paleotemperature history of the Cenozoic and the initiation of Antarctic glaciation: oxygen and carbon isotope analyses in DSDP Sites 277,279 , and 281. In Kennett, J. P., Houtz, R. E., et al., Init. Repts. DSDP, 29: Washington (U.S. Govt. Printing Office), 743-755.

SpieB, V., 1990. Cenozoic magnetostratigraphy of Leg 113 drill sites, Maud Rise, Weddell Sea, Antarctica. In Barker, P. F., Kennett, J. P., et al., Proc. ODP, Sci. Results, 113: College Station, TX (Ocean Drilling Program), 261-315.

Steinmetz, J. C., 1985a. Bibliography and taxa of calcareous nannoplankton-V. INA Newsl., 7:5-28.

1985b. Bibliography and taxa of calcareous nannoplanktonVI. INA Newsl., 7:122-145.

1986. Bibliography and taxa of calcareous nannoplanktonVIII. INA Newsl., 8:66-87.

1987a. Bibliography and taxa of calcareous nannoplanktonIX. INA Newsl., 9:6-29.

1987b. Bibliography and taxa of calcareous nannoplankton-X. INA Newsl., 10:81-109.

1988a. Bibliography and taxa of calcareous nannoplanktonXI. INA Newsl., 10:7-28.

1988b. Bibliography and taxa of calcareous nannoplanktonXII. INA Newsl., 10:60-88.
1989. Bibliography and taxa of calcareous nannoplanktonXIII. INA Newsl., 11:6-23.

Stott, L. D., Kennett, J. P., Shackleton, N. J., and Corfield, R. M., 1990. The evolution of Antarctic surface waters during the Paleogene: inferences from stable isotopic composition of planktonic foraminifers, ODP Leg 113. In Barker, P. F. Kennett, J. P., et al., Proc. ODP, Sci. Results, 113: College Station, TX (Ocean Drilling Program), 849-863.

Vail, P. R., and Hardenbol, J., 1979. Sea level changes during the Tertiary. Oceanus, 22:71-80.

van Andel, T. H., Heath, G. R., and Moore, T. C., 1975. Cenozoic history and paleoceanography of the central equatorial Pacific. Mem. Geol. Soc. Am., 143:1-134.

van Heck, S. E., 1979a. Bibliography and taxa of calcareous nannoplankton. INA Newsl., 1:AB1-B27.

1979b. Bibliography and taxa of calcareous nannoplankton. INA Newsl., 1:ABV1-B42. 1980a. Bibliography and taxa of calcareous nannoplankton. INA Newsl., 2:5-34. 1980b. Bibliography and taxa of calcareous nannoplankton. INA Newsl., 2:43-81.

1981a. Bibliography and taxa of calcareous nannoplankton. INA Newsl., 3:4-41.

1981b. Bibliography and taxa of calcareous nannoplankton. INA Newsl., 3:51-86.

1982a. Bibliography and taxa of calcareous nannoplankton. INA Newsl., 4:7-50. 1982b. Bibliography and taxa of calcareous nannoplankton. INA Newsl., 4:65-96. 1983. Bibliography and taxa of calcareous nannoplankton. INA Newsl., 5:4-13.

Vergnaud Grazzini, C., and Oberhänsli, H., 1986. Isotopic events at the Eocene/Oligocene transition: a review. In Pomerol, C., and Premoli-Silva, I. (Eds.), Terminal Eocene Events: Amsterdam (Elsevier), 311-329.

Wei, W., and Thierstein, H. R., 1991. Upper Cretaceous and Cenozoic calcareous nannofossils of the Kerguelen Plateau (southern Indian Ocean) and Prydz Bay (East Antarctica). In Barron, J., Larsen, B., et al., Proc. ODP, Sci. Results, 119: College Station, TX (Ocean Drilling Program), 467-492.

Wei, W., and Wise, S. W., Jr., 1989. Paleogene calcareous nannofossil magnetobiochronology: results from South Atlantic DSDP Site 516. Mar. Micropaleontol., 14:119-152.

1990a. Middle Eocene-Oligocene calcareous nannoplankton biogeographic gradient of the South Atlantic Ocean. Palaeogeogr., Palaeoclimatol., Palaeoecol., 79:29-61.

1990b. Middle Eocene to Pleistocene calcareous nannofossils recovered by Ocean Drilling Program Leg 113 in the Weddell Sea. In Barker, P. F., Kennett, J. P., et al., Proc. ODP, Sci. Results, 113: College Station, TX (Ocean Drilling Program), 639-666.

, in press a. Correlation of middle Eocene-Oligocene calcareous nannofossil datums with magnetostratigraphy at ODP Site 689 on Maud Rise off East Antarctica. Antarct. J. U.S.

Wei, W., and Wise, S. W., Jr., in press b. Eocene-Oligocene calcareous nan-nofossil magnetobiochronology of the Southern Ocean. Newsl. Stratigr.

Wise, S. W., Jr., 1983. Mesozoic and Cenozoic calcareous nannofossils recovered by Deep Sea Drilling Project Leg 71 in the Falkland Plateau region, Southwest Atlantic Ocean. In Ludwig, W. J., Krasheninnikov, V. A., et al., Init. Repts. DSDP, 71 (Pt. 2): Washington (U.S. Govt. Printing Office), 481-550.

Wise, S. W., Jr., Breza, J. R., Harwood, D. M., and Wei, W., 1991. Paleogene glacial history of Antarctica. In McKenzie, J. A., Müller, D. W., and Weissert, H. (Eds.), Controversies in Modern Geology: London (Academic Press Limited).

Wolfe, J. A., 1971. Tertiary climatic fluctuations and methods of analysis of Tertiary floras. Palaeogeogr., Palaeoclimatol., Palaeoecol., 9:27-57.

1978. A paleobotanical interpretation of Tertiary climates in the Northern Hemisphere. Am. Sci., 66:694-703.

Date of initial receipt: 28 June 1990

Date of acceptance: 11 January 1991

Ms 120B-199 


\section{APPENDIX}

Calcareous Nannofossils Considered in This Paper (in alphabetical order of generic epithets)

Blackites spinosus (Deflandre and Fert) Hay and Towe, 1962

Braarudosphaera bigelowii (Gran and Braarud) Deflandre, 1947 Bramletteius serraculoides Gartner, 1969

Calcidiscus protoannulus (Gartner) Loeblich and Tappan, 1978 Chiasmolithus altus Bukry and Percival, 1971

Chiasmolithus expansus (Bramlette and Sullivan) Gartner, 1970

Chiasmolithus grandis (Bramlette and Riedel) Radomski, 1978.

Chiasmolithus oamaruensis (Deflandre in Deflandre and Fert) Hay, Mohler and Wade, 1966

Chiasmolithus solitus (Bramlette and Sullivan) Locker, 1968

Clausicoccus fenestratus (Deflandre and Fert) Prins, 1979

Coccolithus formosus (Kamptner) Wise, 1973.

Coccolithus pelagicus (Wallich) Schiller, 1930

Cyclicargolithus abisectus (Müller) Wise, 1973

Cyclicargolithus floridanus (Roth and Hay in Hay et al.) Bukry, 1971

Discoaster distinctus Martini, 1958

Discoaster saipanensis Bramlette and Riedel, 1954

Discoaster tanii Bramlette and Riedel, 1954
Discoaster tanii nodifer Bramlette and Riedel, 1954

Isthmolithus recurvus Deflandre in Deflandre and Fert, 1954

Markalius apertus Perch-Nielsen, 1979

Markalius inversus (Deflandre in Deflandre and Fert) Bramlette and Martini, 1964

Neococcolithes dubius (Deflandre in Deflandre and Fert) Black, 1967

Pseudotriquetrorhabdulus inversus (Bukry and Bramlette) Wise in Wise and Constans, 1976

Reticulofenestra bisecta (Hay, Mohler and Wade) Roth, 1970

Reticulofenestra daviesii (Haq) Haq, 1971

Reticulofenestra oamaruensis (Deflandre in Deflandre and Fert) Stradner and Edwards, 1968

Reticulofenestra onusta (Perch-Nielsen) Wise, 1983

Reticulofenestra reticulata (Gartner and Smith) Roth and Thierstein, 1972

Reticulofenestra samodurovii (Hay, Mohler and Wade) Roth, 1970

Reticulofenestra umbilica (Levin) Martini and Ritzkowski, 1968

Sphenolithus moriformis (Brönnimann and Stradner) Bramlette and Wilcoxon, 1967

Triquetrorhabdulus carinatus Martini, 1965

Zygrhablithus bijugatus (Deflandre in Deflandre and Fert) Deflandre, 1959

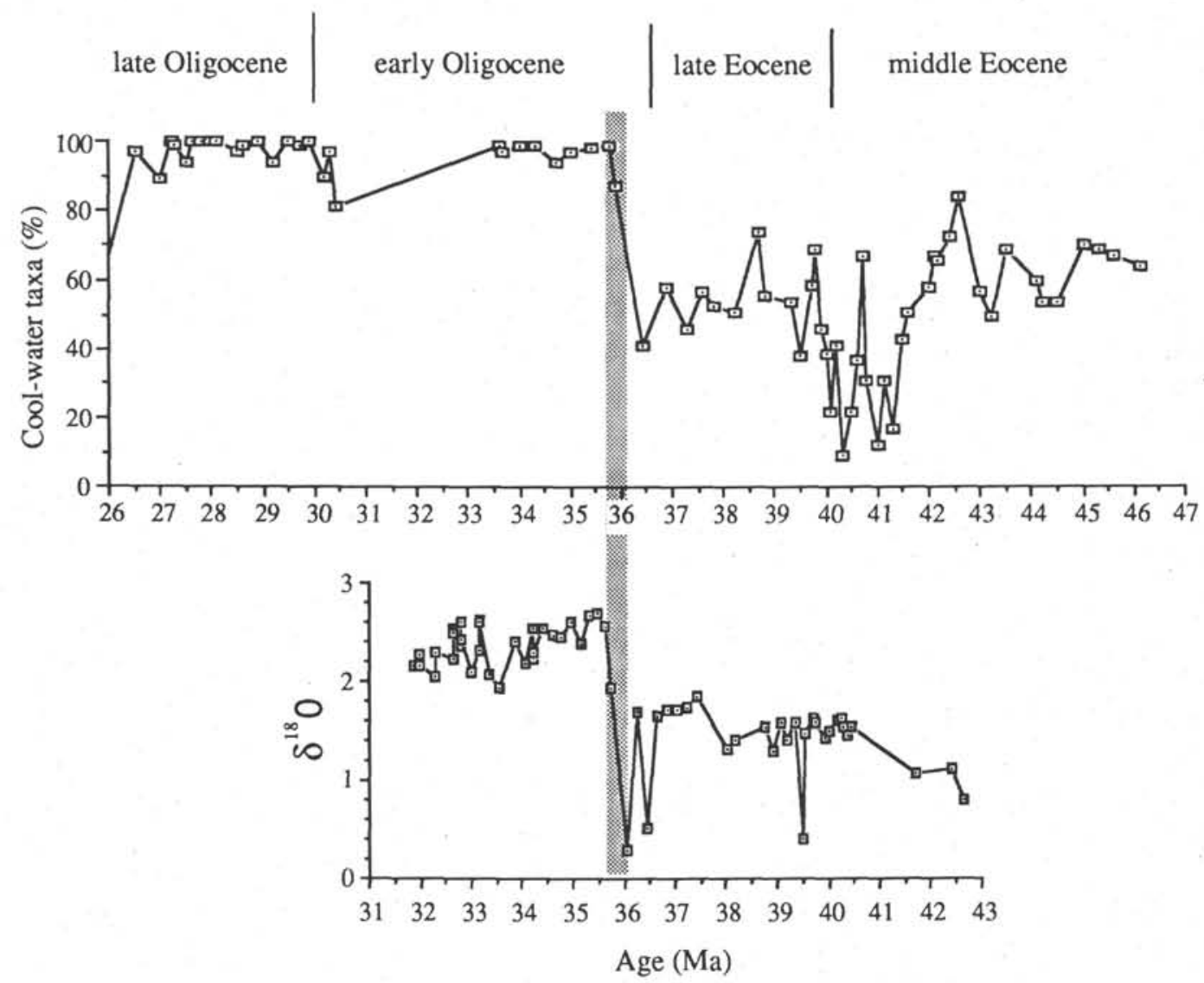

Figure 14. Abundance change of cool-water taxa (chiasmoliths + Isthmolithus recurvus + Reticulofenestra daviesii) through time at Site 689 (modified from Wei and Wise, in press). Changes in the $\delta^{18} \mathrm{O}$ values of planktonic foraminifers (Subbotina angiporoides) through time at the site are shown in the lower panel. Nannofossil data are taken from Wei and Wise (1990a) and isotope data are taken from Stott et al. (1990). Note the abrupt increase in cool-water taxa and the large positive shift in the $\delta^{18} \mathrm{O}$ value of planktonic foraminifers as indicated by the shaded bar. This is a very similar phenomenon as at Site 748 . 